\title{
Decay constants of the heavy-light mesons from the field correlator method
}

\author{
A.M. Badalian ${ }^{a}$, B.L.G. Bakker ${ }^{b}$, and Yu.A. Simonov ${ }^{a}$ \\ a State Research Center \\ Institute of Theoretical and Experimental Physics, \\ Moscow, 117218 Russia \\ $b$ Department of Physics and Astronomy, \\ Vrije Universiteit, Amsterdam
}

(Dated: October 31, 2018)

\begin{abstract}
Meson Green's functions and decay constants $f_{\Gamma}$ in different channels $\Gamma$ are calculated using the Field Correlator Method. Both, spectrum and $f_{\Gamma}$, appear to be expressed only through universal constants: the string tension $\sigma, \alpha_{s}$, and the pole quark masses. For the $S$-wave states the calculated masses agree with the experimental numbers within $\pm 5 \mathrm{MeV}$. For the $D$ and $D_{s}$ mesons the values of $f_{\mathrm{P}}(1 S)$ are equal to $210(10)$ and $260(10) \mathrm{MeV}$, respectively, and their ratio $f_{D_{s}} / f_{D}=1.24(3)$ agrees with recent CLEO experiment. The values $f_{\mathrm{P}}(1 S)=182,216,438 \mathrm{MeV}$ are obtained for the $B, B_{s}$, and $B_{c}$ mesons with the ratio $f_{B_{s}} / f_{B}=1.19(2)$ and $f_{D} / f_{B}=1.14(2)$. The decay constants $f_{\mathrm{P}}(2 S)$ for the first radial excitations as well as the decay constants $f_{\mathrm{V}}(1 S)$ in the vector channel are also calculated. The difference of about $20 \%$ between $f_{D_{s}}$ and $f_{D}, f_{B_{s}}$ and $f_{B}$ directly follows from our analytical formulas.
\end{abstract}

\section{INTRODUCTION}

The decay constants $f_{\mathrm{P}}$ in the pseudoscalar $(\mathrm{P})$ channel, being important characteristics of mesons, in many cases can be directly measured in experiment, and therefore they can provide a precise manner to compare different theoretical approaches and check their accuracy. During the last decade the constants $f_{\mathrm{P}}$ have been studied by many authors in potential models $[1,2,3,4,5,6,7,8]$, in the QCD sum rule method [9, 10], in lattice simulations $[11,12,13,14]$, as well as in experiment $[15,16,17,18,19]$.(The papers $[2,3,4,5,6,7]$ 
contain references and a summary of numerous calculations of decay constants).

The present article is devoted to the systematic derivation of the meson Green's functions in QCD and study of the decay constants for channels with arbitrary quantum numbers $\Gamma$, of which we specifically consider $\mathrm{P}$ and vector $(\mathrm{V})$ channels. For the decay constant $f_{\Gamma}$ transparent analytical expressions will be obtained and in particular, using those, the difference between $f_{D_{s}}$ and $f_{D}, f_{B_{s}}$ and $f_{B}$ can be easily explained.

This paper is an improvement and extension of the earlier paper [20] devoted to the heavy-light (HL) pseudoscalars. Ref. [20] appeared before the systematic formulation of The Field Correlator Method (FCM) [21], in particular, before the derivation of the string Hamiltonian [22], therefore some steps in [20] were not accurately proved. In this paper we give a consistent and general treatment of the meson Green's function and its spectral properties. The main problem, which one encounters when addressing the spectral properties in QCD, is the necessity to include quantitative nonperturbative (NP) methods, which are responsible for the main dynamical phenomena: confinement and chiral symmetry breaking (CSB).

In the FCM, introduced in [23], one derives the effective Hamiltonian, which comprises both confinement and relativistic effects, and contains only universal quantities: the string tension $\sigma$, the strong coupling $\alpha_{\mathrm{s}}$, and the current (pole) quark masses $m_{i}$. We use here the pole quark masses which correspond to the conventional current (Lagrangian) masses $\bar{m}_{q}\left(\bar{m}_{q}\right)$ [16]. The simple local form of this Hamiltonian, which will be called the string Hamiltonian (SH), occurs for objects with temporal scales larger than the vacuum gluon correlation length, $T_{g} \approx 0.2 \mathrm{fm}$, i.e., it is applicable to all QCD bound states with an exception of toponium. Explicit calculations of masses and wave functions with the use of the SH have been done recently for light mesons [24], heavy quarkonia [25], and heavy-light mesons [26], and demonstrate good agreement with experimental masses, leptonic widths, and fine structure effects.

As compared to QCD sum rules and lattice QCD this method has an essential advantage, because the radial and orbital excitations can be considered in this approach on the same grounds as the ground states.

The calculation of spectral coefficients, like decay constants $f_{\Gamma}$, needs an additional step,namely, besides using the SH the computation of all coefficients in the Green's function, including the Dirac spinor structure etc. Moreover, for $\pi$ and $K$ mesons CSB is vitally 
important. Recently the FCM was extended to include the effects of CSB [27], where it was shown that the phenomenon of CSB occurs due to confinement and two characteristic parameters of CSB - the chiral condensate and $f_{\pi}$ - were computed in terms of $\sigma$. The calculation of $f_{\pi}$ and $f_{K}$ can be done using a simple extension of general expression (23), derived in Ref. [27], while here we concentrate on the calculations of the masses and $f_{\Gamma}$ of HL mesons; our method also enables one to calculate $f_{\Gamma}$ for excited states and here decay constants will be calculated for the $2 S$ states.

One important technical problem, which is solved in this paper and allows one to calculate $f_{\Gamma}$ in all channels, is the accurate einbein reformulation of the Fock-Feynman-Schwinger Representation (FFSR), or the world-line representation, where the dynamical quark mass $\omega_{q}$ appears as an integration variable instead of the proper time. The previous step in this direction [22] has enabled one to write only the string Hamiltonian, but the whole Green's function was not attainable. Below in the FFSR we derive the explicit einbein form of the meson Green's function.

The paper is organized as follows: in Section II the general einbein form of the meson Green's function is presented, while in Appendices A - C the details of the derivation are given. In Section III the masses of the heavy-light pseudoscalar mesons are considered. The decay constants are calculated in Section 4, while auxiliary variables are given in Appendices $\mathrm{D}$ and E. Section $\mathrm{V}$ is devoted to the approximations used in our calculations and Section VI contains our concluding remarks.

\section{THE MESON GREEN'S FUNCTION IN THE FFSR}

Here we derive the einbein form of the meson Green's function written as the path integral in the FFSR $[28,29,30]$. We start with the FFSR for the quark Green's function in the gluonic field $A_{\mu}$, which contains both perturbative and NP contributions and Euclidean space-time is assumed everywhere:

$$
\begin{aligned}
S(x, y)= & (m+\hat{D})^{-1}=(m-\hat{D})\left(m^{2}-\hat{D}^{2}\right)^{-1}= \\
& =(m-\hat{D}) \int_{0}^{\infty} d s(D z)_{x y} e^{-K} \Phi_{\sigma}(x, y),
\end{aligned}
$$


with

$$
\begin{aligned}
\hat{D} & =D_{\mu} \gamma_{\mu}, \quad D_{\mu}=\frac{\partial}{\partial x_{\mu}}-i g A_{\mu}, \\
K & =m^{2} s+\frac{1}{4} \int_{0}^{s}\left(\frac{d z_{\mu}}{d \tau}\right)^{2} d \tau \\
\Phi_{\sigma}(x, y) & =P \exp \left(i g \int_{y}^{x} A_{\mu} d z_{\mu}\right) \exp \left(g \int_{0}^{s} \sigma_{\mu \nu} F_{\mu \nu} d \tau\right),
\end{aligned}
$$

where

$$
\begin{aligned}
(D z)_{x y} & =\left.\lim _{N \rightarrow \infty} \prod_{k=1}^{N} \frac{d^{4} \Delta z(k)}{(4 \pi \varepsilon)^{2}}\right|_{\sum_{k} \Delta z(k)=x-y}, N \varepsilon=s \\
\left.\right|_{a=b} & \equiv \int \frac{d^{4} p}{(2 \pi)^{4}} e^{i p(a-b)} .
\end{aligned}
$$

In Eq. (1) the role of the evolution parameter is played by the proper time $s$, whereas in $(D z)_{x y}$ there is an integration over the fourth component $z_{4}(\tau),(0 \leq \tau \leq s)$, which is the Euclidean time of the particle. The crucial point now is to go over in Eq. (1) to the Euclidean time $z_{4} \equiv t$ as an evolution parameter. To get rid of the proper time $s$, one can use the so-called einbein method [31], which was applied to the FFSR in Ref. [22, 30], and here it is developed and used for the correlator of the currents.

To this end the so-called dynamical mass (variable) $\omega(t)$ can be introduced via the relation between the proper time $\tau$ and Euclidean time $t$ :

$$
d \tau=\frac{d t}{2 \omega(t)}, \quad \bar{\omega}=\frac{1}{s} \int_{0}^{s} \omega(\tau) d \tau=\frac{1}{N} \sum_{k=1}^{N} \omega(k)
$$

The integrals in Eq. (1) can now be identically rewritten as

$$
d s(D z)_{x y} \equiv\left(D^{3} z\right)_{\boldsymbol{x} \boldsymbol{y}}(D \omega)=\left.\lim _{N \rightarrow \infty} \prod_{k=1}^{N} \frac{d^{3} \Delta z(k)}{2 \bar{\omega} l^{3}(k)}\right|_{\sum_{k} \Delta \boldsymbol{z}=\boldsymbol{x}-\boldsymbol{y}} \frac{d \omega(k)}{l_{\omega}(k)}
$$

where

$$
l(k)=\sqrt{\frac{2 \pi \Delta t}{\omega(k)}}, \quad l_{\omega}(k)=\sqrt{\frac{2 \pi \omega(k)}{\Delta t}}, \quad N \Delta t=x_{4}-y_{4} \equiv T .
$$

In Appendix A the representations (1), (5) are illustrated by calculating the free quark propagator, where the meaning of $\omega(k)$ and $\bar{\omega}$ in the momentum representation appears to be very simple: $\bar{\omega}=\omega(k)=\sqrt{\boldsymbol{p}^{2}+m^{2}}$. 
We now turn to the meson (quark-antiquark) case and consider the correlator $G_{\Gamma}(x)$ of the currents $j_{\Gamma}(x)$ :

$$
\begin{aligned}
j_{\Gamma}(x) & =\bar{\psi}_{1}(x) \Gamma \psi_{2}(x), \\
\Gamma & =t^{a} \otimes\left(1, \gamma_{5}, \gamma_{\mu}, i \gamma_{\mu} \gamma_{5}\right) \text { for } \mathrm{S}, \mathrm{P}, \mathrm{V}, \text { and A channels, } \\
t^{a} & =\frac{\lambda^{a}}{2}, \quad \operatorname{tr}\left(t^{a} t^{b}\right)=\frac{1}{2} \delta_{a b},
\end{aligned}
$$

and

$$
\begin{aligned}
G_{\Gamma}(x) & \equiv\left\langle j_{\Gamma}(x) j_{\Gamma}(0)\right\rangle_{v} \\
& =4 N_{c} \int Y_{\Gamma}\left(D^{3} z\right)_{x 0}\left(D^{3} \bar{z}\right)_{x 0}\left(D \omega_{1}\right)\left(D \omega_{2}\right) \exp \left(-K_{1}-K_{2}\right) W_{\sigma} .
\end{aligned}
$$

In (8) we have defined the new quantity $Y_{\Gamma}$ :

$$
4 Y_{\Gamma}=\operatorname{tr}_{L}\left(m_{1}-\hat{D}_{1}\right) \Gamma\left(m_{2}-\hat{D}_{2}\right) \Gamma \rightarrow \operatorname{tr}_{L}\left(m_{1}+\omega_{1} \hat{\dot{z}}\right) \Gamma\left(m_{2}-\omega_{2} \hat{\bar{z}}\right) \Gamma,
$$

which can also be written in the operator form:

$$
4 Y_{\Gamma}=\operatorname{tr}_{L}\left(\left(m_{1}-i \hat{p}_{1}\right) \Gamma\left(m_{2}+i \hat{p}_{2}\right) \Gamma\right)
$$

with $p_{\mu}^{(i)}\left(\hat{p}_{i}=p_{\mu} \gamma_{\mu}\right)$ - the momentum of particle $i$ as it is derived in Appendix B for the convenience of the reader ( $\operatorname{tr}_{L}$ means the trace over Dirac indices). The resulting expressions for $Y_{\Gamma}$ in the $\mathrm{V}, \mathrm{A}, \mathrm{S}$, and $\mathrm{P}$ channels are given below in Eqs. (35).

In (8) the symbol $W_{\sigma}=\Phi_{\sigma_{1}}(x, y) \Phi_{\sigma_{2}}(y, x)$ stands for the average value of the Wilson loop with the insertions of the operator

$$
\Lambda_{\sigma}^{(1)} \equiv \exp \left(g \sigma_{\mu \nu}^{(1)} \int_{0}^{s} F_{\mu \nu} d \tau\right)=\exp \left(g \sigma_{\mu \nu}^{(1)} \int_{0}^{T} F_{\mu \nu}\left(z\left(t_{1}\right)\right) \frac{d t_{1}}{2 \omega_{1}\left(t_{1}\right)}\right)
$$

for the quark line and of the operator $\Lambda_{\sigma}^{(2)}$ for the antiquark line:

$$
\Lambda_{\sigma}^{(2)} \equiv \exp \left(-g \sigma_{\mu \nu}^{(2)} \int_{0}^{s} F_{\mu \nu} d \tau\right)=\exp \left(-g \sigma_{\mu \nu}^{(2)} \int_{0}^{T} F_{\mu \nu}\left(\bar{z}\left(t_{2}\right)\right) \frac{d t_{2}}{2 \omega_{2}\left(t_{2}\right)}\right) .
$$

Since in HL mesons we will consider spin-effects as a perturbation, in the first approximation both factors $\Lambda_{\sigma}^{(1)}$ and $\Lambda_{\sigma}^{(2)}$ are replaced by 1 and $W_{\sigma}$ is simplified.

In Appendix $\mathrm{C}$ one can find all explicit steps for the derivation of the correlator (8) in the simplest case when the gluon interaction is absent, i.e., for $W_{\sigma} \equiv 1$. One can see there that the quark-loop contribution is reconstructed with the correct coefficients. Now we turn to the case when the NP interaction is included in $W_{\sigma}$. 
In general, $W_{\sigma}$ contains all effects of the interaction which include: (i) the perturbative static gluon exchange; (ii) the radiative corrections to $G_{\Gamma}$ in the form of the operator anomalous dimension and corrections to $f_{\Gamma}$; (iii) the NP contributions to $G_{\Gamma}$. To calculate all of them one can use the background perturbation theory [32, 33] and the FCM. For all hadrons of interest to us (with a size larger than $T_{g} \approx 0.2 \mathrm{fm}$ ) the use of the FCM reduces to the appearance of the area-law factor in $W_{\sigma}$, which is accompanied by Coulomb, radiative, and spin-dependent factors. (For more discussion see the reviews [30, 34]). As was shown in Refs. $[33,34]$ this produces (apart from radiative corrections) the local interaction $\hat{V}(r)$, which for not so large angular momentum $(L \leq 4)$ can be presented as $W_{\sigma}=\exp \left(-\int_{0}^{T} d t \hat{V}\right)$, where the interaction,

$$
\hat{V}(r)=V_{0}(r)+V_{\mathrm{SD}}+\Delta V_{\text {string }}+V_{\mathrm{SE}},
$$

contains the static potential:

$$
V_{0}(r)=\sigma r-\frac{4}{3} \frac{\alpha_{\mathrm{st}}(r)}{r},
$$

and the spin-dependent part $V_{\mathrm{SD}}(r)$ given by:

$$
V_{\mathrm{SD}}=V_{\mathrm{SS}}+V_{\mathrm{LS}}+V_{\mathrm{T}}
$$

with spin-spin, spin-orbit, and tensor terms; the self-energy contribution $V_{\mathrm{SE}}$ [35], and also a "string correction" (occurring only for the states with $L \neq 0[22,24]$ ). For the $S$-wave mesons, considered here,

$$
\hat{V}(r)=V_{0}(r)+V_{\mathrm{SS}}+V_{\mathrm{SE}}
$$

while for the spin-averaged masses only two terms are left in the potential, $V(r)=V_{0}(r)+$ $V_{\mathrm{SE}}$. In Section $\mathrm{V}$ we shall also take into account radiative corrections and the operator anomalous dimension.

One can now rewrite $G_{\Gamma}$ in Eq. (8), separating c.m. and relative distance coordinates,

$$
\boldsymbol{\eta}=\Delta \boldsymbol{z}_{1}-\Delta \boldsymbol{z}_{2}, \quad \boldsymbol{\rho}=\frac{\omega_{1} \Delta \boldsymbol{z}_{1}+\omega_{2} \Delta \boldsymbol{z}_{2}}{\omega_{1}+\omega_{2}}, \quad \omega_{\mathrm{r}}=\frac{\omega_{1} \omega_{2}}{\omega_{1}+\omega_{2}}
$$

where all coordinates are labelled with the index $k$ as in Eq. (6).

Integrating out the c.m. coordinate $d \boldsymbol{\rho}$ and $d \omega_{+}=\omega_{1}+\omega_{2}$, as shown in Appendix C, one arrives at the path integral in the relative coordinate $d \boldsymbol{\eta}$, which can be expressed through the $\mathrm{SH}$. Indeed, from the path integral formalism [30, 36] it is known that a general equivalence 
relation holds:

$$
\int \frac{\left(D^{3} \eta\right)_{x y}}{\left(2 \pi \Delta t / \omega_{\mathrm{r}}(k)\right)^{3 / 2}} e^{-\sum_{k}\left(\frac{\omega_{\mathrm{r}}(k) \eta^{2}(k)}{2 \Delta t}+\hat{V}(k) \Delta t\right)}=\left\langle x\left|e^{-\hat{H} T}\right| y\right\rangle,
$$

where

$$
\hat{H}=\frac{\boldsymbol{p}_{\eta}^{2}}{2 \omega_{\mathrm{r}}}+\hat{V}(\eta), \quad \boldsymbol{p}_{\eta}=\frac{1}{i} \frac{\partial}{\partial \boldsymbol{\eta}} .
$$

Taking into account the integral (see Appendix C):

$$
\int \frac{2 d \omega_{\mathrm{r}}(k)}{\sqrt{\omega_{\mathrm{r}}(k)} \sqrt{\frac{2 \pi}{\Delta t}}} e^{-2 \omega_{\mathrm{r}}(k) \Delta t-\frac{\left(p^{2}+m^{2}\right) \Delta t}{2 \omega_{\mathrm{r}}(k)}}=e^{-2 \sqrt{p^{2}+m^{2}} \Delta t},
$$

one obtains the important relation:

$$
\int G_{\Gamma}(x) d^{3} \boldsymbol{x}=N_{c}\left\langle 0\left|\frac{Y_{\Gamma}}{\bar{\omega}_{1} \bar{\omega}_{2}} e^{-\hat{H} T}\right| 0\right\rangle,
$$

where the Hamiltonian is

$$
\hat{H}=\sqrt{\boldsymbol{p}^{2}+m_{1}^{2}}+\sqrt{\boldsymbol{p}^{2}+m_{2}^{2}}+\hat{V}(r) .
$$

Then with the use of the spectral expansion the expression (21) can be presented as

$$
\left\langle 0\left|\frac{Y_{\Gamma}}{\bar{\omega}_{1} \bar{\omega}_{2}} e^{-\hat{H} T}\right| 0\right\rangle=\sum_{n} \frac{\left\langle Y_{\Gamma}\right\rangle_{n}\left|\varphi_{n}(0)\right|^{2}}{\left\langle\bar{\omega}_{1}\right\rangle_{n}\left\langle\bar{\omega}_{2}\right\rangle_{n}} e^{-M_{n} T},
$$

where $\varphi_{n}$ and $M_{n}$ are the eigenfunction (e.f.) and the eigenvalue (e.v.) of the Hamiltonian (22). It follows from the extremum conditions (D.7) that the variables $\bar{\omega}_{1}$ and $\bar{\omega}_{2}$ in Eq. (21) are defined as the operators,

$$
\bar{\omega}_{i}=\sqrt{\boldsymbol{p}^{2}+m_{i}^{2}},
$$

while in Eq. (19) $\left\langle\bar{\omega}_{i}\right\rangle_{n}$ is the matrix element over this operator for the $n L$ state.

On the other hand for the l.h.s. of Eq. (21) one can also use the conventional spectral decomposition:

$$
\begin{aligned}
\int G_{\Gamma}(x) d^{3} \boldsymbol{x} & =\sum_{n} \int d^{3} \boldsymbol{x}\left\langle 0\left|j_{\Gamma}\right| n\right\rangle\left\langle n\left|j_{\Gamma}\right| 0\right\rangle e^{i \boldsymbol{P} \cdot \boldsymbol{x}-M_{n} T} \frac{d^{3} \boldsymbol{P}}{2 M_{n}(2 \pi)^{3}} \\
& =\sum_{n} \varepsilon_{\Gamma} \otimes \varepsilon_{\Gamma} \frac{\left(M_{n} f_{\Gamma}^{n}\right)^{2}}{2 M_{n}} e^{-M_{n} T} .
\end{aligned}
$$

Here we have used the standard definition for $f_{\Gamma}^{n} \equiv f_{\Gamma}(n S)$ :

$$
\left\langle 0\left|j_{\Gamma}\right| n, \boldsymbol{P}=0\right\rangle=\varepsilon_{\Gamma} M_{n} f_{\Gamma}^{n},
$$


where $\varepsilon_{\Gamma}=\varepsilon_{\mu}^{(k)}$ for $\mathrm{V}$ and A channels, $\varepsilon_{\Gamma}=1$ for $\mathrm{S}$ and $\mathrm{P}$ channels, while the polarization vector $\varepsilon_{\mu}^{(k)}$ satisfies the normalization condition:

$$
\sum_{k=1,2,3} \varepsilon_{\mu}^{(k)}(q) \varepsilon_{\nu}^{(k)}(q)=\delta_{\mu \nu}-\frac{q_{\mu} q_{\nu}}{q^{2}}
$$

Inserting the expression (23) into (21) and using the relation (25), one obtains the decay constant $f_{\Gamma}^{n}$ in the channel $\Gamma$ for the $n S$ state:

$$
\left(f_{\Gamma}^{n}\right)^{2}=\frac{2 N_{c}\left\langle Y_{\Gamma}\right\rangle\left|\varphi_{n}(0)\right|^{2}}{\left\langle\bar{\omega}_{1}\right\rangle_{n}\left\langle\bar{\omega}_{2}\right\rangle_{n} M_{n}}=\frac{6\left|\varphi_{n}(0)\right|^{2}}{M_{n}} \frac{\left\langle Y_{\Gamma}\right\rangle}{\left\langle\bar{\omega}_{1}\right\rangle_{n}\left\langle\bar{\omega}_{2}\right\rangle_{n}} .
$$

To derive the relations (23) and (28) we have used the essential property of the SH Eq. (22) that the average value $\left\langle\omega_{i}(k)\right\rangle$ of the operator $\omega_{i}(k)$ is equal to the average of the operator $\sqrt{\boldsymbol{p}^{2}+m_{i}^{2}}$, and hence the average $\bar{\omega}_{i}=\frac{1}{N} \sum_{k=1}^{N} \omega_{i}(k)$, denoted as $\left\langle\bar{\omega}_{i}\right\rangle_{n}$ is

$$
\left\langle\bar{\omega}_{i}\right\rangle_{n}=\left\langle\omega_{i}(k)\right\rangle_{n}=\left\langle\sqrt{\boldsymbol{p}^{2}+m^{2}}\right\rangle_{n}
$$

where the average is assumed to be taken over the eigenstate $\varphi_{n}(r)$ :

$$
\left\langle\bar{\omega}_{i}\right\rangle_{n}=\left\langle\varphi_{n}\left|\sqrt{\boldsymbol{p}^{2}+m_{i}^{2}}\right| \varphi_{n}\right\rangle
$$

In the most general case this average may differ from the path-integral average $\left\langle\bar{\omega}_{i}\right\rangle$ in Eqs. (4), (5), (8). However, the analysis of this problem, done in Ref. [37], shows that the difference between the two definitions is small ( $\lesssim 3 \%$ for the lowest states) and we assume here that the same accuracy holds for our basic relation (28).

In Eq. (28) the mass of the ${ }^{1} S_{0}$-wave meson $M_{n}\left({ }^{1} S_{0}\right)=M_{\mathrm{on}}-\frac{3}{4} \Delta_{\mathrm{HF}}+\Delta_{\mathrm{SE}}$, where $M_{\mathrm{on}}$ is the e.v. of the Hamiltonian (22) with the static interaction (14):

$$
\begin{aligned}
H_{0} & =\sqrt{m_{1}^{2}+\boldsymbol{p}^{2}}+\sqrt{m_{2}^{2}+\boldsymbol{p}^{2}}+V_{0}(r), \\
H_{0} \varphi_{n} & =M_{\mathrm{on}} \varphi_{n} .
\end{aligned}
$$

The general expression of the self-energy correction to the meson mass $M(n S)$ is calculated in [35] (see Appendix E) (it comes from the NP contributions to the quark and antiquark mass):

$$
\Delta_{\mathrm{SE}}=\sum_{i=1,2}\left\{\left(-\frac{1.5 \sigma \eta_{f_{i}}}{\pi\left\langle\bar{\omega}_{i}\right\rangle}\right)+\frac{\sigma^{2}}{4\left\langle\bar{\omega}_{i}\right\rangle\left[m_{i}+\left\langle\bar{\omega}_{i}\right\rangle / 2+\varepsilon(\tilde{\omega})\right]^{2}}\right\},
$$

where the factor $\eta_{f}(i)$ depends on the flavor of the $i$-th quark (antiquark) and its analytical expressions (E.2) are deduced in Ref. [35]. For the $u(d), s, c$, and $b$ quarks the values

$$
\eta_{u(d)}=1.0, \quad \eta_{s}=0.65, \quad \eta_{c}=0.35, \quad \eta_{b}=0.03
$$


are obtained in Appendix E ${ }^{1}$.

It is worthwhile to notice that for the $b$ quark $\eta_{b}$ is small and its contribution to $\Delta_{\mathrm{SE}}$ is small $(\sim 1 \mathrm{MeV})$ and can be neglected. Therefore, for the $B, B_{s}$, and $B_{c}$ mesons we have to use in Eq. (32) only a contribution which comes from the lighter quark (antiquark) $q_{1}\left(\bar{q}_{1}\right)$ denoted later by the index 1 . For the $D$ and $D_{s}$ mesons both terms (with $i=1$ and $i=2$ ) are taken into account, although the contribution from the c-quark term is small, around $-20 \mathrm{MeV}$.

Thus, the scheme of our calculations of $f_{\Gamma}$ is as follows:

1. First, one calculates the e.v. $M_{\text {on }}$ of the SH (22) with the static interaction (14) and then takes into account the self-energy (32) and the HF corrections:

$$
M_{n}\left({ }^{3} S_{1}\right)=M_{\mathrm{on}}+\Delta_{\mathrm{SE}}+\frac{1}{4} \Delta_{\mathrm{HF}}, \quad M_{n}\left({ }^{1} S_{0}\right)=M_{\mathrm{on}}+\Delta_{\mathrm{SE}}-\frac{3}{4} \Delta_{\mathrm{HF}} .
$$

2. The values $\left\langle\bar{\omega}_{1}\right\rangle$ and $\left\langle\bar{\omega}_{2}\right\rangle$ in Eqs (28) and (32), are the matrix elements (m.e.) of the kinetic energy term defined in Eq. (24).

3. The factor $\left\langle Y_{\Gamma}\right\rangle$ (in the channel $\Gamma$ ) can be computed in terms of the momenta of a quark and an antiquark, or in the c.m. system in terms of the relative momentum $\boldsymbol{p}$, with the following results for the m.e. $\left\langle Y_{\Gamma}\right\rangle$ (see Appendix B):

$$
\begin{aligned}
& \left\langle Y_{\mathrm{V}}\right\rangle=m_{1} m_{2}+\left\langle\bar{\omega}_{1}\right\rangle\left\langle\bar{\omega}_{2}\right\rangle+\frac{1}{3}\left\langle\boldsymbol{p}^{2}\right\rangle, \\
& \left\langle Y_{\mathrm{P}}\right\rangle=m_{1} m_{2}+\left\langle\bar{\omega}_{1}\right\rangle\left\langle\bar{\omega}_{2}\right\rangle-\left\langle\boldsymbol{p}^{2}\right\rangle=\left\langle Y_{A_{4}}\right\rangle .
\end{aligned}
$$

Here we use for the operators $Y_{\Gamma}$ the notations:

$$
\begin{aligned}
\hat{Y}_{\mathrm{V}} & =\frac{1}{3} \sum_{i} \operatorname{tr}\left[\left(m_{1}-\hat{D}_{1}\right) \gamma_{i}\left(m_{2}-\hat{D}_{2}\right) \gamma_{i}\right], \\
\hat{Y}_{\mathrm{P}}=\hat{Y}_{A_{4}} & =-\left[\operatorname{tr}\left(m_{1}-\hat{D}_{1}\right) \gamma_{4} \gamma_{5}\left(m_{2}-\hat{D}_{2}\right) \gamma_{4} \gamma_{5}\right] .
\end{aligned}
$$

In the case of the $\mathrm{P}$ channel with both $m_{1}, m_{2} \rightarrow 0$ due to CSB there appears an additional mass term in Eq. (35), which can be computed through field correlators. (The $\pi$ and $K$ mesons will be considered later [27]).

For the calculations of $\left\langle Y_{\Gamma}\right\rangle$ one needs also to know the m.e. $\left\langle\boldsymbol{p}^{2}\right\rangle_{n S}$ and the w.f. at the origin, $\varphi_{n}(0)=R_{n}(0) / \sqrt{4 \pi}$; they are given in Appendix D.

\footnotetext{
${ }^{1}$ Note, that these values of $\eta_{f(i)}$ in (33) are not fitting parameters, but calculated through the same input $m_{i}$ and $\sigma$
} 
4. Our calculations are done with the relativistic $\mathrm{SH}(22)$ which was derived in einbein approximation (EA), therefore the w.f. at the origin must also be calculated with the use of the EA (see Appendix D), which provides an accuracy of $\lesssim 5 \%[26,37]$. In the nonrelativistic limit, $m_{i} \gg \sqrt{\sigma}$, one can easily find that $\left\langle\bar{\omega}_{i}\right\rangle \approx m_{i}$, while $\left\langle\boldsymbol{p}^{2}\right\rangle \sim O(\sigma)$ can be neglected in Eqs. (35), and therefore

$$
\begin{aligned}
\left\langle Y_{\mathrm{V}}\right\rangle_{\mathrm{NR}} & \approx 2 m_{1} m_{2}+O(\sigma), \quad\left\langle Y_{S}\right\rangle_{\mathrm{NR}} \approx O(\sigma) \\
\left\langle Y_{A_{i}}\right\rangle_{\mathrm{NR}} & \approx O(\sigma), \quad\left\langle Y_{A_{4}}\right\rangle_{\mathrm{NR}}=2 m_{1} m_{2}+O(\sigma), \\
\left\langle Y_{\mathrm{P}}\right\rangle_{\mathrm{NR}} & =2 m_{1} m_{2}+O(\sigma) .
\end{aligned}
$$

Thus in the nonrelativistic limit for $f_{\Gamma}^{(n)}$ in the $\mathrm{V}$ and $\mathrm{P}$ channels one obtains the well-known result [1]:

$$
\left(f_{\Gamma}^{n}\right)_{\mathrm{NR}}^{2}=\frac{4 N_{c}}{M_{n}}\left|\varphi_{n}(0)\right|^{2} \quad(\Gamma=V, P),
$$

while in the $S$ channel $f_{S} \rightarrow 0$.

5. As a final step one needs to compute the radiative corrections to $f_{\Gamma}^{n}$, which come from the short-distance (large momentum) perturbative gluon contributions. Neglecting interference terms they can be written as in [20, 30],

$$
\left\langle W_{\sigma}\right\rangle=\left\langle W_{\mathrm{OGE}}\right\rangle\left\langle W_{\text {nonpert }}\right\rangle
$$

with

$$
\left\langle W_{\mathrm{OGE}}\right\rangle=Z_{m} \exp \left(-\frac{4}{3 \pi} \iint \frac{d z_{4} d z_{4}^{\prime} \alpha_{\mathrm{s}}\left(z-z^{\prime}\right)}{\left(z-z^{\prime}\right)^{2}}\right),
$$

where $Z_{m}$ is a regularization factor. After separating the Coulomb interaction in $\hat{H}$ in this way, one gets the correction to $\left\langle W_{\sigma}\right\rangle$, and $f_{\Gamma}^{2}$ can be written in the form:

$$
f_{\Gamma}^{2} \rightarrow \xi_{\Gamma} f_{\Gamma}^{2}, \quad \xi_{\Gamma}=1+c_{\Gamma} \alpha_{\mathrm{s}}+O\left(\alpha_{\mathrm{s}}^{2}\right)
$$

Another important contribution from perturbative gluon exchanges (GE) is the account of Asymptotic Freedom (AF) in the coupling constant $\alpha_{\mathrm{s}}$ in (40), which is especially important for the value of $\varphi_{n}(0)$ in the $S$-wave channels. In our calculations we use the GE interaction where in the strong coupling $\alpha_{B}(r)$ the AF behavior is taken into account. Nevertheless, it is of interest to compare the w.f. at the origin with and without AF behavior in the GE term, introducing the factor $\rho_{A F}=\left|\varphi_{n}^{(\mathrm{AF})}(0) / \varphi_{n}(0)\right|^{2}$, which appears to be around 0.80 for the $1 S$ 
TABLE I: The pole quark masses $m_{q}$ (pole) (in $\mathrm{GeV}$ ), used in this paper, and the constituent masses $m_{q}^{\mathrm{C}}$ from $[2,4,5]$.

\begin{tabular}{|l|c|c|c|c|c|}
\hline quark & $\mathrm{b}$ & $\mathrm{c}$ & $\mathrm{s}$ & $\mathrm{u}$ & $\mathrm{d}$ \\
\hline$m_{q}$ (pole) this paper & 4.78 & 1.40 & 0.180 & 0.005 & 0.008 \\
\hline$m_{q}$ from $[4]$ & 4.655 & 1.511 & 0.216 & 0.071 & 0.071 \\
\hline$m_{q}^{\mathrm{C}}$ from $[2]$ & 4.977 & 1.628 & 0.419 & 0.220 & 0.220 \\
\hline$m_{q}^{\mathrm{C}}$ from $[5]$ & 5.158 & 1.755 & 0.535 & 0.371 & 0.377 \\
\hline
\end{tabular}

states [20]. Then $f_{\Gamma}^{2}$ (with AF taken into account) can be expressed through $\tilde{f}_{\Gamma}^{2}$ (where AF is neglected) as $f_{\Gamma}^{2}=\tilde{f}_{\Gamma}^{2} \xi_{\Gamma} \rho_{\mathrm{AF}}$.

6. We conclude this section with the discussion of the input parameters $m_{i}, \alpha_{\mathrm{s}}$ and $\sigma$. We take $\sigma=0.18 \mathrm{GeV}^{2}$ for all HL mesons (as in light mesons and in heavy quarkonia [38]); $m_{i}$ are the conventional pole masses which are defined through the Lagrangian (current) masses in the $\overline{M S}$-scheme (see $[16,39]$ and references therein):

$$
m_{i}=\bar{m}_{\overline{M S}}\left(\bar{m}_{\overline{M S}}\right)\left\{1+\frac{4}{3} \frac{\alpha_{\mathrm{s}}\left(\bar{m}_{\overline{M S}}\right)}{\pi}+\eta_{2}\left(\frac{\alpha_{\mathrm{s}}}{\pi}\right)^{2}+O\left(\alpha_{\mathrm{s}}\right)^{3}\right\} .
$$

We use here the pole masses presented in Table I. They correspond to the conventional current masses $\bar{m}_{c}=1.18 \mathrm{GeV}$ and $\bar{m}_{b}=4.20 \mathrm{GeV}$ while for the strange quark the pole mass, $m_{s}=180 \mathrm{MeV}$ is taken at the scale $\mu \approx 0.5 \mathrm{GeV}\left(r_{0} \approx 0.5 \mathrm{fm}\right)$ and therefore it should be larger than the standard $m_{s}(2 \mathrm{GeV}) \approx 100 \mathrm{MeV}$ [16]. For the $u$ and $d$ quarks $m_{u}=5$ $\mathrm{MeV}, m_{d}=8 \mathrm{MeV}$ are taken.

One can see the essential difference between the input masses in our SH and the constituent masses used in the relativistic instantaneous Bethe-Salpeter Method [5], and also in the spinless Salpeter equation [2]. The $b, c$, and $s$ quark masses from [4], where the relativistic Dirac Hamiltonian is used for a light quark, are not large and can be considered as the pole masses, while $m_{u}=m_{d}=71 \mathrm{MeV}$ seem to be too large.

7. The coupling $\alpha_{\mathrm{st}}(r)$ in the GE term is taken here as the vector coupling $\alpha_{B}(r)$ in background perturbation theory (in two-loop approximation) from [39]. At large distances this theory predicts saturation: $\alpha_{B}(r) \rightarrow \alpha_{\text {crit }}=$ const (this coupling $\alpha_{B}(r)$ is in good 
agreement with lattice potential at small distances [40]):

$$
\begin{aligned}
& \alpha_{B}(r)=\frac{2}{\pi} \int_{0}^{\infty} d q \frac{\sin q r}{q} \alpha_{B}(q), \\
& \alpha_{B}(q)=\frac{4 \pi}{\beta_{0} t_{B}}\left\{1-\frac{\beta_{1}}{\beta_{0}^{2}} \frac{\ln t_{B}}{t_{B}}\right\}, \quad t_{B}=\ln \frac{q^{2}+M_{B}^{2}}{\Lambda_{B}^{2}} .
\end{aligned}
$$

Here the background mass $M_{B}=1 \mathrm{GeV}$ can be expressed through the $\sqrt{\sigma}$ [38], while the QCD constant $\Lambda_{B}$ is given by

$$
\Lambda_{B}=\Lambda_{\overline{M S}} \exp \left(\frac{\frac{31}{3}-\frac{10}{9} n_{f}}{2 \beta_{0}}\right) .
$$

It is of interest to notice that in our calculations of HL meson properties the value $n_{f}=3$ is strongly preferable.

In this way the problem is uniquely defined and no fitting parameters are introduced. The resulting masses and decay constants of HL mesons appear to be unbiased theoretical predictions which will be compared with recent experiments, lattice data, and other theoretical predictions.

\section{MASSES OF HEAVY-LIGHT MESONS}

In the relativistic $\mathrm{SH}$ a correct choice of the static interaction $V_{0}(r)$, which defines the e.v. of the unperturbed Hamiltonian $H_{0}(31)$, is of great importance. Here, for all HL mesons we take the static potential from $[38,39]$ :

$$
V_{B}(r)=\sigma r-\frac{4}{3} \frac{\alpha_{B}(r)}{r}
$$

with $\sigma=0.18 \mathrm{GeV}^{2}$, the number of flavors $n_{f}=3$, the vector coupling $\alpha_{B}(r)$ (43) also contains

$$
\Lambda_{B}\left(n_{f}=3\right)=360 \mathrm{MeV}, \quad M_{B}=1.0 \mathrm{GeV} .
$$

For this choice the saturated (critical) value of the vector coupling, reached at large $r$, is equal $\alpha_{\text {crit }}\left(n_{f}=3\right)=0.495$, while at $r \approx r_{0}=0.5 \mathrm{fm}, \alpha_{B}\left(r_{0}\right) \cong 0.43$.

It is very convenient to start the calculations with the spin-averaged masses $M_{\operatorname{cog}}(n L)$,

$$
\begin{aligned}
& M_{\mathrm{cog}}(n S)=M_{0}(n S)+\Delta_{\mathrm{SE}}(n S), \\
& M_{\mathrm{cog}}(n L)=M_{0}(n L)+\Delta_{\mathrm{SE}}(n L)+\Delta_{\mathrm{str}}(n L) \quad(L \neq 0),
\end{aligned}
$$


TABLE II: The spin-averaged masses $M_{\operatorname{cog}}(1 S)(\text { in } \mathrm{MeV})^{a)}$.

\begin{tabular}{|l|l|l|l|l|}
\hline Meson multiplet & $M_{0}(1 S)$ & $\Delta_{\mathrm{SE}}$ & $M_{\mathrm{cog}}(1 S)$ & $M_{\mathrm{cog}}(\exp )^{b)}$ \\
\hline$D-D^{*}$ & 2139 & -164 & 1975 & $1974.8(4)$ \\
$D_{s}-D_{s}^{*}$ & 2177 & -105 & 2072 & $2076.0(6)$ \\
$B-B^{*}$ & 5433 & -120 & 5313 & $5313.5(6)$ \\
$B_{s}-B_{s}^{*}$ & 5468 & -72 & 5396 & $5400.7(32)$ \\
$B_{c}-B_{c}^{*}$ & 6332 & -17 & 6315 & - \\
\hline
\end{tabular}

a) The $\Delta_{\text {SE }}$ are calculated in Appendix E.

b) The experimental numbers are taken from PDG [16] and [41].

since these masses do not depend on any additional parameter. In contrast to $M_{\operatorname{cog}}$ the masses of the singlet and triplet states depend also on the parameters defining the HF interaction, or for states with $L \neq 0$ the masses $M\left(J^{P C}\right)$ depend also on the coupling $\alpha_{\mathrm{FS}}\left(\mu_{\mathrm{FS}}\right)$, which defines the fine-structure splittings, and these couplings are not well determined. In Eq. (47) $M_{0}(n L)$ is the e.v. of Eq. (31) (see Tables II,V). The self-energy term $\Delta_{\mathrm{SE}}(n L)(32)$ and the string correction $\Delta_{\text {str }}(n L)=\left\langle H_{\text {str }}\right\rangle$ (D.12) are defined by analytical expressions and discussed in Appendices D and E.

The calculated $M_{\operatorname{cog}}(1 S)$, as seen from Table II, agree with the experimental numbers with an accuracy better than $5 \mathrm{MeV}$. It is of interest to notice that the difference $M_{\operatorname{cog}}\left(D_{s}^{+}\right)-M_{\operatorname{cog}}\left(D^{+}\right)$, which in experiment is $99 \mathrm{MeV}$, is only partly due to dynamical reasons: $M_{0}\left(D_{s}\right)-M_{0}(D)=38 \mathrm{MeV}$, but mostly occurs due to the difference in the SE contributions, equal to $60 \mathrm{MeV}$.

With the use of our number $M_{\operatorname{cog}}\left(B_{c}\right)=6315 \mathrm{MeV}$ and $M\left(B_{c}\right)_{\exp }=6275(7) \mathrm{MeV}[41]$ one can predict the mass of the vector state $B_{c}^{*}\left(1^{3} S_{1}\right)$ :

$$
M\left(B_{c}^{*}\right)=6328(7) \mathrm{MeV}, \text { if } M\left(B_{c}\right)=6275(7) \mathrm{MeV} .
$$

The masses of the triplet and singlet HL mesons are calculated taking into account the HF interaction which in general contains both perturbative and NP contributions:

$$
\Delta_{\mathrm{HF}}(n S)=\Delta_{\mathrm{HF}}^{\mathrm{P}}+\Delta_{\mathrm{HF}}^{\mathrm{NP}}
$$


where the $\mathrm{P}$ term with the one-loop correction is taken from [42]:

$$
\Delta_{\mathrm{HF}}^{\mathrm{P}}(n S)=\frac{8}{9} \frac{\alpha_{\mathrm{HF}}(\mu)}{\omega_{1} \omega_{2}}\left|R_{n}(0)\right|^{2}\left(1+\frac{\alpha_{\mathrm{HF}}}{\pi} \rho\right) .
$$

Here $\rho=\frac{5}{12} \beta_{0}-\frac{8}{3}-\frac{3}{4} \ln 2$ and for $n_{f}=3, \rho\left(n_{f}=3\right)=0.5635$, so that the one-loop correction is about $6 \%$. From here on for simplicity we use the notation $\omega_{1}, \omega_{2}$ instead of $\left\langle\bar{\omega}_{1}\right\rangle_{n},\left\langle\bar{\omega}_{2}\right\rangle_{n}$.

The NP contribution to the HF splitting was derived in [43] and with good accuracy it is given by the m.e.:

$$
\Delta_{\mathrm{HF}}^{\mathrm{NP}}(n S) \approx 1.20 \frac{\pi^{2}}{18} \frac{G_{2}}{\omega_{1} \omega_{2}}\left\langle r K_{1}\left(\frac{r}{T_{g}}\right)\right\rangle_{n S} .
$$

Here $G_{2}$ is the gluonic condensate, for which we take the value $G_{2}=0.043 \mathrm{GeV}^{4}$ [43], which provides the correct value of the string tension $\sigma=0.18 \mathrm{GeV}^{2}$. For the $D$ and $D_{s}$ mesons the NP term (51) appears to be not small (as well as for the $J / \psi-\eta_{c}$ splitting): around $10 \mathrm{MeV}$ if the gluonic (vacuum) correlation length is $T_{g}=0.2 \mathrm{fm}$ [44]. However, at present the value of $T_{g}$ is not known with high accuracy and $T_{g}=0.28 \mathrm{fm}$ was obtained from unquenched lattice data [45]. Due to this uncertainty in $T_{g}$ we have two possibilities to describe the HF effects in HL mesons:

A. In the case of $T_{g} \approx 0.2 \mathrm{fm}, \Delta_{\mathrm{HF}}^{\mathrm{NP}} \approx 10 \mathrm{MeV}$ is not large. Then in the perturbative term, Eq. (50), we need to take a rather large coupling: $\alpha_{\mathrm{HF}}=0.40$ for the $D$ and $D_{s}$ mesons and $\alpha_{\mathrm{HF}}=0.32$ for the $B$ and $B_{s}$ mesons, to reach agreement with experiment.

B. In the case of large gluonic length, $T_{g}=0.3 \mathrm{fm}$, the NP contribution $\Delta_{\mathrm{HF}}^{\mathrm{NP}}$ appears to be larger, about $22 \mathrm{MeV}$, and therefore the coupling $\alpha_{\mathrm{HF}}$ can be taken smaller. In this case for the $D$ and $D_{s}$ mesons the value $\alpha_{\mathrm{HF}}=0.365$ (rather close to that for the $J / \Psi-\eta_{c}$ splitting [46]) gives rise to agreement with the experimental HF splitting. In Table III the calculated HF splittings both for $T_{g}=0.2 \mathrm{fm}$ and $T_{g}=0.3 \mathrm{fm}$ are given.

Taking the HF splittings from Table 3 the masses of the singlet and triplet states can be calculated; they are presented in Table IV $\left(M_{\operatorname{cog}}(1 S)\right.$ are taken from Table II).

The calculated triplet and singlet masses for the ground states appear to be in good agreement with the experimental numbers (with an accuracy $\lesssim 5 \mathrm{MeV}$ ). In our analysis we have observed that for the $D$ and $D_{s}$ mesons the value $M_{\operatorname{cog}}(1 S)$ is very sensitive to the pole mass of the $c$ quark and high accuracy can be reached only for $m_{c}$ (pole) $=1.40 \mathrm{GeV}$; at the same time we cannot exclude that for the $B_{c}$ meson the choice of $m_{c}=1.38 \mathrm{GeV}$ is also possible. 
TABLE III: The hyperfine splittings (in MeV) in HL mesons. Case A: $T_{g}=0.20 \mathrm{fm}, \alpha_{\mathrm{HF}}=0.40$ for the $D$ and $D_{s}$ mesons and $\alpha_{\mathrm{HF}}=0.32$ for the $B$ and $B_{s}$ mesons. Case B: $T_{g}=0.30 \mathrm{fm}$, $\alpha_{\mathrm{HF}}=0.365$ for the $D$ and $D_{s}$ mesons and $\alpha_{\mathrm{HF}}=0.305$ for the $B$ and $B_{s}$ mesons.

\begin{tabular}{|l|l|l|l|l|}
\hline & \multicolumn{4}{|c|}{$T_{g}=0.20 \mathrm{fm}$} \\
\hline Multiplet & $\Delta_{\mathrm{HF}}^{\mathrm{P}}$ & $\Delta_{\mathrm{HF}}^{\mathrm{NP}}$ & $\Delta_{\mathrm{HF}}$ (tot) & $\Delta_{\mathrm{HF}}(\exp )$ \\
\hline$D^{*}-D$ & 131.6 & 8.9 & 140.5 & $140.64 \pm 0.10$ \\
$D_{s}^{*}-D_{s}$ & 130.7 & 8.9 & 139.6 & $143.8 \pm 0.4$ \\
$B^{*}-B$ & 43.5 & 1.5 & 45.0 & $45.78 \pm 0.35$ \\
$B_{s}^{*}-B_{s}$ & 44.2 & 1.4 & 45.8 & $44.2 \pm 1.8$ \\
\hline & \multicolumn{5}{|c|}{$T_{g}=0.30 \mathrm{fm}$} \\
\hline$D^{*}-D$ & 119.4 & 21.8 & 141.2 & $140.6 \pm 0.1$ \\
$D_{s}^{*}-D_{s}$ & 118.6 & 21.6 & 140.2 & $143.8 \pm 0.4$ \\
$B^{*}-B$ & 41.3 & 3.7 & 45.0 & $45.8 \pm 0.4$ \\
$B_{s}-B$ & 42.0 & 3.4 & 45.4 & $44.2 \pm 1.8$ \\
\hline
\end{tabular}

TABLE IV: The masses (in MeV) of the ground states (for $T_{g}=0.3 \mathrm{fm}$ ).

\begin{tabular}{|l|l|l|l|l|l|l|l|l|}
\hline & $D^{ \pm}$ & $D^{* \pm}$ & $D_{s}$ & $D_{s}^{*}$ & $B$ & $B^{*}$ & $B_{s}$ & $B_{s}^{*}$ \\
\hline this paper & 1869.1 & 2010.3 & 1966.8 & 2107.1 & 5279.3 & 5324.2 & 5362.0 & 5407.4 \\
\hline Exper. $^{a)}$ & 1869.3 & 2010.0 & 1968.2 & 2112.0 & 5279.0 & 5325.0 & 5367.7 & 5411.7 \\
& \pm 0.4 & \pm 0.4 & \pm 0.4 & \pm 0.6 & \pm 0.5 & \pm 0.6 & \pm 1.8 & \pm 3.2 \\
\hline
\end{tabular}

a) The experimental numbers are taken from PDG [16] and $M\left(B_{s}^{*}\right)$ from Ref. [19].

For the $B_{c}$ meson, with the use of $\alpha_{\mathrm{HF}}=0.26$ and $M_{\mathrm{cog}}=6313 \mathrm{MeV}$ from Table II, we obtain $\Delta_{\mathrm{HF}}\left(B_{c}\right)=44 \mathrm{MeV}$, which gives $M\left(B_{c}\right)=6280 \mathrm{MeV}$ close to $M\left(B_{c}\right)(\exp )=6275(7)$ $\mathrm{MeV}[42]$ and $M\left(B_{c}^{*}\right)=6324 \mathrm{MeV}$.

Finally, in Table $\mathrm{V}$ we give the calculated masses $M(2 S)$ for the first radial excitations, which are not yet found in experiment. Our prediction for the singlet and triplet masses of the radially excited HL mesons strongly depends on the value of $\alpha_{\mathrm{HF}}$ taken. If for the $D(2 S)$ and $D_{s}(2 S)$ mesons one takes the value $\alpha_{\mathrm{HF}}\left(\mu_{2}\right)=0.30$, as in the case of $\eta_{c}(2 S)[46]$, 
TABLE V: The masses $M_{\mathrm{cog}}(2 S), M\left(2^{1} S_{0}\right)$ and $M\left(2^{3} S_{1}\right)$ (in $\left.\mathrm{MeV}\right)$ for heavy-light mesons $\left(\alpha_{\mathrm{HF}}=\right.$ $0.30)$.

\begin{tabular}{|l|l|l|l|l|l|l|}
\hline & & $D(2 S)$ & $D_{s}(2 S)$ & $B(2 S)$ & $B_{s}(2 S)$ & $B_{c}(2 S)^{a)}$ \\
\hline this paper & $M_{0}(2 S)$ & 2758 & 2797 & 5998 & 6034 & 6868 \\
& $M_{\operatorname{cog}}(2 S)$ & 2615 & 2702 & 5888 & 5966 & 6852 \\
& $M\left(2^{1} S_{0}\right)$ & 2560 & 2646 & 5864 & 5941 & 6821 \\
& $M\left(2^{3} S_{1}\right)$ & 2633 & 2721 & 5896 & 5974 & 6862 \\
\hline from Ref. [4] & $M\left(2^{1} S_{0}\right)$ & 2589 & 2700 & 5886 & 5985 & \\
& $M\left(2^{3} S_{1}\right)$ & 2692 & 2806 & 5920 & 6019 & \\
\hline from Ref. [2] & $M\left(2^{1} S_{0}\right)$ & 2580 & 2670 & 5900 & 5980 & 6855 \\
& $M\left(2^{3} S_{1}\right)$ & 2640 & 2730 & 5930 & 6010 & 6887 \\
\hline
\end{tabular}

a) For the $B_{c}(2 S)$ meson we use the value $\alpha_{\mathrm{HF}}=0.26$.

then the values $M\left(2^{1} S_{0}\right)$ and $M\left(2^{3} S_{1}\right)$, given in Table V, are obtained.

The triplet and singlet masses, calculated here, are rather close to those from [2, 4], nevertheless for the $2^{1} S_{0}$ states our numbers are systematically lower by $\sim 30-50 \mathrm{MeV}$.

In this paper we do not consider orbital excitations of HL mesons, it will be done in our next paper. Still, we would like to notice that our values of $M\left(B_{s 1}\right)$ and $M\left(B_{s 2}\right)$ lie in the region 5.82 - 5.83, i.e., approximately by $100 \mathrm{MeV}$ lower than in Ref. [4] and close to the recent experimental data [19].

\section{DECAY CONSTANTS OF HEAVY-LIGHT MESONS}

The general formula for the decay constants $f_{\Gamma}^{2}(23)$ can be rewritten (later on we shall use the notation $\omega_{1}, \omega_{2}$ for $\left.\left\langle\omega_{1}\right\rangle,\left\langle\omega_{2}\right\rangle\right)$ as follows

$$
\left(f_{\Gamma}^{n}\right)^{2}(n S)=\frac{3\left\langle Y_{\Gamma}\right\rangle}{2 \pi \omega_{1} \omega_{2} M_{n}}\left|R_{n}(0)\right|^{2},
$$

which contains the w.f. at the origin $R_{n}(0)=\varphi_{n}(0) / \sqrt{4 \pi}$, the average kinetic energies $\omega_{1}, \omega_{2}$, the meson mass $M_{n}=M(n S)$, and also the m.e. $\left\langle\boldsymbol{p}^{2}\right\rangle$ in the factor $\left\langle Y_{\Gamma}\right\rangle$. All these auxiliary values are given in Appendix D (Tables IX and X). Then the values of $f_{\mathrm{P}}$ can be easily calculated. They are given in Table VI, together with the experimental, unquenched 
TABLE VI: The decay constants $f_{\mathrm{P}}$ (in $\mathrm{MeV}$ ).

\begin{tabular}{|l|l|l|l|l|l|}
\hline & $D$ & $D_{s}$ & $B$ & $B_{s}$ & $B_{c}$ \\
\hline Ref. [20] & 206 & 252 & 174 & - & - \\
Ref. [7] & $230(25)$ & $248(27)$ & $196(29)$ & $216(32)$ & $322(42)$ \\
Ref. [6] & 234 & 268 & 189 & 218 & - \\
\hline lattice [11, 14] & $235(22)$ & $266(28)$ & - & $206(10)$ & - \\
quenched & & & & & \\
\hline lattice [11, 13] & $201(20)$ & $249(19)$ & $216(38)$ & $259(32)$ & $440(2)$ \\
$n_{f}=2+1$ & & & & & \\
\hline this paper & $210(10)$ & $260(10)$ & $182(8)$ & $216(8)$ & $438(10)$ \\
\hline experiment & $222.6(20)^{a)}$ & $280(23)^{a)}$ & $\left.160_{-80}^{+50} b\right)$ & & \\
& & & $229(70)^{c)}$ & & \\
\hline
\end{tabular}

a) The experimental values are taken from Ref. [17].

b) BaBar data [18]

c) Belle data [18]

and quenched lattice data, and some other theoretical analyses.

From Table VI one can see that our central value of $f_{B_{d}}$ is $15 \%$ smaller than the one in unquenched lattice QCD [13], but rather close to $f_{B}$ in relativistic models [6, 7].

For the analysis of experimental data on direct measurements of the leptonic decay, $P \rightarrow l \nu$, it is important to know the ratios of the decay constants, which in our calculations are,

$$
\frac{f_{D_{s}}}{f_{D}}=1.24(3), \quad \frac{f_{B_{s}}}{f_{B}}=1.19(3), \quad \frac{f_{D_{s}}}{f_{B_{s}}}=1.20(3) ; \quad \frac{f_{D}}{f_{B}}=1.15(2) .
$$

These ratios are in good agreement with recent lattice data (unquenched) [13, 15] and close to the experimental number obtained by the CLEO collaboration with $f_{D_{s}} / f_{D}=1.27(14)$ [17]. It is of interest to compare these ratios with other theoretical calculations, which are typically smaller than our numbers, and also with recent lattica data (see Table VII).

Due to the large theoretical errors in the ratios $\zeta_{D}=f_{D_{s}} / f_{D}$ and $\zeta_{B}=f_{B_{s}} / f_{B}$ (see Table VII) one cannot judge what is the true value of $\zeta_{D}$ and $\zeta_{B}$, however, from our general 
TABLE VII: The ratios $f_{D_{s}} / f_{D}, f_{B_{s}} / f_{B}$, and $f_{D_{s}} / f_{B_{s}}$.

\begin{tabular}{|l|l|l|l|}
\hline & $f_{D_{s}} / f_{D}$ & $f_{B_{s}} / f_{B}$ & $f_{D_{s}} / f_{B_{s}}{ }^{a}$ \\
\hline RPM [6] & 1.15 & 1.15 & 1.23 \\
\hline BS [7] & $1.08(1)$ & $1.10(1)$ & $1.15(1)$ \\
\hline $\begin{array}{l}\text { lattice [13] } \\
\text { unquenched }\end{array}$ & $1.24(8)$ & $1.20(4)$ & $1.01(8)$ \\
\hline this work & $1.24(3)$ & $1.19(3)$ & $1.20(3)$ \\
\hline experiment & $1.27(14)$ & & \\
\hline
\end{tabular}

a) The ratio of the central values is taken for $f_{D_{s}}$ and $f_{B_{s}}$.

formula (52) for $f_{\mathrm{P}}$ it follows that $f_{D_{s}}$ and $f_{D}$ as well as $f_{B_{s}}$ and $f_{B}$ have to differ by $\sim 20 \%$. This happens due to the presence in Eq. (52), through $\left\langle Y_{\Gamma}\right\rangle$, of a term proportional to $m_{1} m_{2}$ : $m_{1}=0.008 \mathrm{GeV}$ for $D^{+}$and $m_{1}=0.18 \mathrm{GeV}$ for $D_{s}^{+}, m_{2}=1.40$ in both cases. If one neglects this term then the value $f_{D_{d}}=f_{D_{u}}=208 \mathrm{MeV}$ practically does not change, while for the $D_{s}$ meson one obtains the essentially smaller number $215 \mathrm{MeV}$, instead of $f_{D_{s}}=260 \mathrm{MeV}$ in the case with $m_{1}=m_{s}=0.18 \mathrm{GeV}$. Thus, the ratios $\zeta_{D}$ and $\zeta_{B}$ appear to be very sensitive to what one takes for the pole mass of the $s$ quark and can be used as a convenient criterium to choose $m_{s}$ at low renormalization scale. Note that the factor $\left|R_{1}(0)\right|^{2} /\left(\omega_{1} \omega_{2}\right)=0.35(1)$ turns out to be the same both for the $D$ and $D_{s}$ mesons. Our number for $\zeta_{D}=1.24(3)$ (with $\left.m_{s}=0.18 \mathrm{GeV}\right)$ is in agreement with the experimental number $\zeta_{D}(\exp )=1.27(14)$ [17].

The first radial excitations of HL mesons are considered here neglecting open channels which can decrease the w.f. at the origin. In this approximation

$$
\begin{aligned}
& f_{\mathrm{P}}(D(2 S))=167 \mathrm{MeV}, f_{\mathrm{P}}\left(D_{s}(2 S)\right)=201 \mathrm{MeV}, \\
& f_{\mathrm{P}}(B(2 S))=168 \mathrm{MeV}, f_{\mathrm{P}}\left(B_{s}(2 S)\right)=194 \mathrm{MeV}, \quad f_{\mathrm{P}}\left(B_{c}(2 S)\right)=347 \mathrm{MeV} .
\end{aligned}
$$

From these numbers it follows that for the $2 S$ states $f_{D_{s}} / f_{D}=1.20$ and $f_{B_{s}} / f_{B}=1.15$ are changed only by about $\approx 4 \%$, although the absolute values of $f_{\mathrm{P}}(2 S)$ appear to be $\approx 20 \%$ smaller than for the ground states.

One can compare our results for $f_{\mathrm{P}}$ with other theoretical calculations (see Table VI). The agreement with potential-model results $[6,7]$ is evident with the only exception for $f_{\mathrm{P}}\left(B_{c}\right)$ : our number $f_{\mathrm{P}}\left(B_{c}\right)=439 \mathrm{MeV}$ is about $30 \%$ higher, and is close to the one calculated in 
TABLE VIII: The vector decay constants $f_{\mathrm{V}}$ (in $\mathrm{MeV}$ ) and the ratio $f_{\mathrm{V}} / f_{\mathrm{P}}$ for the $1 S$ states in heavy-light mesons.

\begin{tabular}{|l|l|l|l|l|l|}
\hline & $D^{*}$ & $D_{s}^{*}$ & $B^{*}$ & $B_{s}^{*}$ & $B_{c}^{*}$ \\
\hline$\left\langle\boldsymbol{p}^{2}\right\rangle$ in $\mathrm{GeV}^{2}$ & 0.273 & 0.291 & 0.359 & 0.383 & 0.784 \\
\hline$f_{\mathrm{V}}[5]$ & $340(23)$ & $375(24)$ & $238(18)$ & $272(20)$ & $418(24)$ \\
\hline$f_{\mathrm{V}}$, this work & $273(13)$ & $307(18)$ & $200(10)$ & $230(12)$ & $453(20)$ \\
\hline$f_{\mathrm{V}} / f_{\mathrm{P}}[5]$ & $1.48(26)$ & $1.51(26)$ & $1.21(27)$ & $1.26(28)$ & $1.30(24)$ \\
\hline$f_{\mathrm{V}} / f_{\mathrm{P}}$ this paper & $1.27(5)$ & $1.17(4)$ & $1.08(4)$ & $1.07(4)$ & $1.03(3)$ \\
\hline
\end{tabular}

unquenched lattice QCD, where $f_{\mathrm{P}}\left(B_{c}\right)$ (lattice) $=420(20) \mathrm{MeV}$ in [13]. A detailed analysis of $f_{B_{c}}$ (in the framework of the potential model and QCD sum rule approach with radiative corrections taken into account) [47] gives the value $f_{B_{c}} \approx 400 \mathrm{MeV}$, which is approximately $10 \%$ lower than our number.

Finally, some remarks about decay constants in V channels. As seen from the expressions for $\left\langle Y_{\mathrm{V}}\right\rangle$ and $\left\langle Y_{\mathrm{P}}\right\rangle, f_{\mathrm{V}}^{2}$ has to be larger than $f_{\mathrm{P}}^{2}$ (since $M_{\mathrm{V}}$ and $M_{\mathrm{P}}$ are close to each other), while $\left\langle\boldsymbol{p}^{2}\right\rangle$ enters with different signs

$$
\frac{f_{\mathrm{V}}^{2}(n S)}{f_{\mathrm{P}}^{2}(n S)}=\frac{\left(m_{1} m_{2}+\omega_{1} \omega_{2}+\frac{1}{3}\left\langle\boldsymbol{p}^{2}\right\rangle_{n S}\right)}{\left(m_{1} m_{2}+\omega_{1} \omega_{2}-\left\langle\boldsymbol{p}^{2}\right\rangle_{n S}\right)} \frac{M_{\mathrm{P}}(n S)}{M_{\mathrm{V}}(n S)}>1 .
$$

These ratios (for the ground states) are given in Table VIII. For the heavy $B_{c}$ meson this ratio is approaching unity.

From Table VIII one can see that our numbers for the vector decay constants are systematically lower than $f_{\mathrm{V}}$ (central values) from Ref. [5]: by $\approx 20 \%$ for the $D$ and $D_{s}$ mesons and by $\approx 15 \%$ for the $B$ and $B_{s}$ mesons, although their values lie within the large theoretical errors.

\section{APPROXIMATIONS}

We discuss here the approximations we made and the accuracy of our results. The starting expression for the current correlator (8) is exact, because the FFSR is an exact representation of the meson Green's function. The main approximation refers to the transition from the FFSR path-integral to the local Hamiltonian formalism (19) neglecting quark pair and hybrid 
production and taking spin-dependent interactions as a perturbation. The accuracy of this approximation is determined by several factors:

1) Neglect of hybrid excitations in the Wilson loop, which actually leads to a multichannel Hamiltonian. The hybrid admixture was shown to be small, of the order of few percent for ground state mesons $[48,49]$.

2) The use of the EA to define the w.f. at the origin and some m.e. The corresponding accuracy was checked in [37] and shown to be around $5 \%$.

One should stress that the SH contains only well defined fundamental parameters: the pole quark masses, $\Lambda_{\mathrm{QCD}}$, and the universal string tension $\sigma$, and does not contain any fitting parameters, in particular, there is no overall constant often used in the static potential, or in the mass. The accuracy of the SH was checked for light mesons [24], heavy quarkonia [25], heavy-light mesons [26], glueballs [50], and hybrids [49]. In all cases meson masses are in agreement with experimental and lattice data with an accuracy of a few percent.

Concerning the decay constants $f_{\mathrm{P}}$, a special sensitivity occurs in the w.f. at the origin $\left|\varphi_{n}(0)\right|^{2}$, i.e., to the behaviour of the GE potential (or the vector coupling $\alpha_{\text {st }}(r)$ ) at small distances, not only in the AF region $(r \lesssim 0.1 \mathrm{fm})$ but also in the region $0.1 \mathrm{fm} \lesssim r \lesssim 0.3$ fm, which in [39] called the intermediate region. This behavior is known quite well for the perturbative part, where $\Lambda_{\mathrm{QCD}}$ is well known (for $n_{f}=5 \Lambda_{\overline{M S}}^{(5)}=217_{-23}^{+25} \mathrm{MeV}$ [16]). The major uncertainty comes from two sources:

(i) the behaviour of the spin-dependent part, in particular, the HF interaction, where smearing of the $\delta$ function can drastically change the wave function for systems of small size, $R \lesssim 0.4 \mathrm{fm}$. For HL mesons with $R \gtrsim 0.6 \mathrm{fm}$ this effect is becoming less important (for more discussion of the influence of the HF interaction see [51]).

(ii) We estimate the accuracy of the resulting $\left|\varphi_{n}(0)\right|^{2}$ better than $\pm 5 \%$. Thus the accuracy of our computed values of $f_{\mathrm{P}}$ is expected to be $\lesssim 8 \%$, while the ratio of decay constant has better accuracy, $\lesssim 4 \%$.

At this point one should discuss the effects which were unaccounted for till now. First of all, this concerns the radiative corrections due to transverse gluon exchanges and higher loops. These corrections contain the (pseudo) evolution factor, governed by the operator anomalous dimension, and considered in [52] two decades ago,

$$
X_{M}=\left(\frac{\alpha_{s}\left(m_{b}\right)}{\alpha_{s}\left(m_{c}\right)}\right)^{-\frac{6}{33-2 n_{f}}} \equiv x^{\frac{6}{33-2 n_{f}}}
$$


One can estimate that when going from $B$ to $D$ mesons this factor changes only by $4 \%$.

Nowadays the radiative corrections are done within HQET and known to three-loop accuracy [53], e.g. for $f_{D}$ one has

$$
f_{D}=\frac{1}{\sqrt{m_{c}}}\left[1+c_{1} \frac{\alpha_{s}^{(4)}\left(m_{c}\right)}{4 \pi}+c_{2}^{(3)}\left(\frac{\alpha_{s}^{(4)}\left(m_{c}\right)}{4 \pi}\right)^{2}+\ldots\right] F^{(3)}\left(m_{c}\right)+O\left(\frac{\Lambda_{\mathrm{QCD}}}{m_{c}}\right)
$$

where $F^{(3)}\left(m_{c}\right)$ is subject to the operator anomalous dimension correlations, and $c_{1}=-2 C_{f}$ etc. As a result, e.g. for the ratio $f_{B} / f_{D}$, one has [53]

$$
\frac{f_{B}}{f_{D}}=\sqrt{\frac{m_{c}}{m_{b}}} X_{M}\left[1+r_{1}(x-1) \frac{\alpha_{s}^{(4)}\left(m_{b}\right)}{4 \pi}+O\left(\left(\frac{\alpha_{s}^{(4)}}{4 \pi}\right)^{2}\right)\right]+O\left(\alpha_{s}^{3}, \frac{\Lambda_{\mathrm{QCD}}}{m_{c, b}}\right)
$$

with $r_{1}=\frac{56}{75} \zeta_{2}+\frac{4403}{1875} \cong 3.58$, and $x \approx 1.56$. One can see that loop corrections contribute less than $4 \%$ and can be neglected within the accuracy of HQET and our approximations.

From Eq. (58) it follows that in HQET the ratio $f_{B} / f_{D}<1$ as it happens in relativistic models $[2,5,6,7]$ and also in our calculations where $f_{B} / f_{D} \approx 0.87(2)$, while in unquenched lattice data this ratio is larger than unity, but has large computational error $(\sim 20 \%)$.

We now turn again to Table VI and discuss our results in comparison to other calculations. The first important point, which should be stressed, is that our input is minimal and fundamental, e.g. the pole masses in Table I correspond to the current masses quoted by PDG [16]. This is in contrast to many relativistic potential models with spinless Salpeter [2] or Bethe-Salpeter Hamiltonian [5, 7], where the constituent quark masses are used as input. In addition an overall constant is usually introduced in the interaction. In many respects our approach can be considered on the same grounds as lattice simulations, or the QCDsum-rule approach (where instead of $\sigma$ several additional condensates are used). Moreover, the advantage of our approach is that excited states can be considered as well as the ground states. For excited states a quark (antiquark) of a given $n L$ state has its characteristic "constituent" mass $\langle\bar{\omega}\rangle_{n L}$, which grows for higher $n L$. Due to this effect, and also to the negative string correction, in our approach the masses of the radial and orbital excitations appear to be smaller $(\sim 20-40 \mathrm{MeV})$ than in other relativistic models (see Table V).

Looking at Table VI one notices a good agreement (within $6-10 \%$ ) of our results with unquenched lattice data and experiment (for $f_{D}$ and $f_{D_{s}}$ ). Thus one can conclude that the Field Correlator Method and its essential part, the effective String Hamiltonian, appears to be successful in the prediction of $f_{\Gamma}$ as well as in other tests done so far $[21,22,23,24,25,26]$. 


\section{CONCLUSIONS}

In this paper we have studied the current correlator $G_{\Gamma}(x)=\left\langle j_{\Gamma}(x) j_{\Gamma}(0)\right\rangle$ and the integral $J_{\Gamma}=\int G_{\Gamma}(x) d \boldsymbol{x}$ in an arbitrary channel $\Gamma$ with the use of the FFS path-integral representation. This method allows one to express decay constants $f_{\Gamma}(n S)$ for HL mesons through well-defined characteristics of the relativistic SH which was successfully used before in light mesons and heavy quarkonia.

It is essential that the SH does not contain any fitting parameters, being fully defined by universal fundamental values: the conventional pole masses, the string tension $\sigma$, and the strong vector coupling $\alpha_{\mathrm{st}}(r)$.

The analytical expressions, obtained here for $f_{\mathrm{P}}$, show that the decay constants $f_{D_{s}}$ and $f_{B_{s}}$ for the ground states have to be always larger by 20-25\% than $f_{D}$ and $f_{B}$ because of the large difference between the pole (current) masses of the strange and light $u(d)$ quarks. This theoretical statement is supported by recent experimental data [17].

In our analysis we have observed that

- The calculated masses $M\left(1^{1} S_{0}\right)$ and $M\left(1^{3} S_{1}\right)$ of all HL mesons agree with the experimental numbers within $\pm 5 \mathrm{MeV}$. Our prediction for $B_{c}^{*}$ is $M\left(B_{c}^{*}\right)=6325(10)$ $\mathrm{MeV}$.

- The calculated masses of the first radial excitations $M\left(2^{1} S_{0}\right)$ for the $D$ and $D_{s}$ mesons appear to be $\approx 40 \mathrm{MeV}$ lower and for the $B, B_{s}$, and $B_{c}$ mesons $\approx 20-30 \mathrm{MeV}$ lower than the numbers from Refs. [2, 4].

- For the decay constants the values $f_{D}=210(10) \mathrm{MeV}$ and $f_{D_{s}}=260(10) \mathrm{MeV}$ are obtained. Their ratio $f_{D_{s}} / f_{D}=1.24(3)$ is close to the experimental number $1.27(14)$ $[17]$.

- Our decay constants $f_{B}=182(8) \mathrm{MeV}$ and $f_{B_{s}}=216(8) \mathrm{MeV}$ give the ratio $f_{B_{s}} / f_{B}=$ $1.19(3)$ which agrees with a recent unquenched lattice number $[11,13]$.

- In the $\mathrm{V}$ channel the ratio $f_{\mathrm{V}} / f_{\mathrm{P}}$ is monotonically decreasing while going from the $D$ meson to the heavier mesons: it is equal to 1.27(6), 1.17(4), 1.08(4), 1.07(4), 1.03(3) for the $D, D_{s}, B, B_{s}$, and $B_{c}$ mesons, respectively. For the $D_{s}^{*}$ and $B_{s}^{*}$ mesons calculated here, $f_{\mathrm{V}}$ turned out to be $\approx 20 \%$ smaller than in Ref. [5]. 


\section{APPENDIX A: FREE QUARK PROPAGATOR IN THE EINBEIN PATH-INTEGRAL REPRESENTATION}

One starts with the FFSR for the free quark propagator, which can be written as

$$
S(x, y)=(m-\hat{\partial}) \int_{0}^{\infty} d s(D z)_{x y} \exp (-K)
$$

and introduces the einbein variable, or the dynamical mass, $\omega(t)$ as in $(4)$, such that the function $K$ can be rewritten as

$$
\begin{aligned}
K & =m^{2} s+\frac{1}{4} \int_{0}^{s}\left(\frac{d z_{\mu}(\tau)}{d \tau}\right)^{2} d \tau \\
& =\int_{0}^{T} d t\left\{\frac{m^{2}}{2 \omega(t)}+\frac{\omega(t)}{2}+\frac{\omega(t)}{2}\left(\frac{d z_{i}(t)}{d t}\right)^{2}\right\}
\end{aligned}
$$

In $(D z)_{x y}$, Eq. (3), there is an integration over the time components of the path, namely,

$$
\left(D z_{4}\right) \equiv \prod_{k} \frac{d \Delta z_{4}(k)}{(4 \pi \varepsilon)^{1 / 2}} \delta\left(\sum \Delta z_{4}-T\right)
$$

where $T \equiv x_{4}-y_{4}$. With the use of (4) one can rewrite the integration element in (A.3) as follows $\left(t \equiv z_{4}\right)$,

$$
\frac{d \Delta z_{4}(k)}{\sqrt{4 \pi \varepsilon}}=2 d \omega(k) \sqrt{\frac{\varepsilon}{4 \pi}}=\frac{d \omega(k) \sqrt{\Delta t}}{\sqrt{2 \pi \omega(k)}}, \sqrt{\varepsilon}=\sqrt{\frac{\Delta t}{2 \omega(k)}} .
$$

Moreover, the $\delta$-function in (A.3) acquires the form

$$
\delta\left(\sum \Delta z_{4}-T\right)=\delta(2 \bar{\omega} s-T)
$$

where we have defined

$$
\bar{\omega}=\frac{1}{s} \int_{0}^{s} \omega(\tau) d \tau
$$

As a result in (A.1) one can integrate over $d s$ using the $\delta$-function (A.5), and rewrite $d s(D z)_{x y}$ as it is shown in Eq. (5) of the main text.

Then one can write the Green's function as follows:

$$
S(x, y)=(m-\hat{\partial}) \int \prod \frac{d^{3} \Delta z_{i}(k)}{l^{3}} e^{-K} \frac{d \omega(k)}{l_{\omega}(k)} \frac{d^{3} p}{(2 \pi)^{3}} e^{i \boldsymbol{p} \cdot\left(\boldsymbol{x}-\boldsymbol{y}-\sum \Delta \boldsymbol{z}(k)\right)},
$$

where $K$ is given in (A.2), and $l, l_{\mu}$ in Eq. (6). 
The integration over $d^{3} \Delta z_{i}(k)$ yields

$$
S(x, y)=(m-\hat{\partial}) \int \frac{d^{3} p}{(2 \pi)^{3}} e^{i \boldsymbol{p}(\boldsymbol{x}-\boldsymbol{y})-\frac{1}{2} \int_{0}^{T} d t \omega(t)\left(1+\frac{p^{2}+m^{2}}{\omega^{2}(t)}\right)} \frac{1}{2 \bar{\omega}}(D \omega) .
$$

Taking into account the relation,

$$
\int_{0}^{\infty} \frac{d \omega(k)}{\sqrt{\omega(k)}} e^{-\frac{\Delta t}{2}\left(\omega(k)+\frac{p^{2}+m^{2}}{\omega(k)}\right)}=\sqrt{\frac{2 \pi}{\Delta t}} e^{-\Delta t \sqrt{p^{2}+m^{2}}}
$$

one has for the scalar part $G(x, y)$, defined by $S=(m-\hat{\partial}) G$, the following expression:

$$
G(x, y)=\int \frac{d^{3} p}{(2 \pi)^{3}} \frac{e^{i \boldsymbol{p} \cdot(\boldsymbol{x}-\boldsymbol{y})-\int_{0}^{T} d t \sqrt{\boldsymbol{p}^{2}+m^{2}}}}{2 \sqrt{\boldsymbol{p}^{2}+m^{2}}}
$$

where we have used the relation following from the stationary point in the integral (A.9):

$$
\bar{\omega}=\frac{1}{s} \int_{0}^{s} \omega(\tau) d \tau=\frac{1}{N} \sum_{k=1}^{N} \omega(k)=\sqrt{\boldsymbol{p}^{2}+m^{2}} .
$$

The expression (A.10) can be compared with the conventional integral,

$$
G(\boldsymbol{r}, T)=\int \frac{d^{4} p}{(2 \pi)^{4}} \frac{e^{i \boldsymbol{p} \cdot \boldsymbol{r}+i p_{4} T}}{p_{4}^{2}+\boldsymbol{p}^{2}+m^{2}}, \quad \boldsymbol{r}=\boldsymbol{x}-\boldsymbol{y},
$$

which reduces to (A.10) after integrating over $d p_{4}$ for $T>0$. The expression (A.12) is the standard form of the free propagator.

\section{APPENDIX B: CALCULATION OF THE FACTOR $\left\langle Y_{\Gamma}\right\rangle$}

Here we use and extend Appendix 1 of Ref. [20], considering the quark propagator (1) in the gluonic field $A_{\mu}: S(x, y) \equiv\left[m-\gamma_{\mu}\left(\partial / \partial x_{\mu}-i g A_{\mu}\right)\right] G(x, y)$. The derivative $\partial / \partial x_{\mu}$, acting on $G(x, y)$, differentiates only the $\delta$-function, $\delta\left(x_{\mu}-y_{\mu}-\sum_{k=1}^{N} \Delta z_{\mu}\right)$ and can be rewritten as a derivative in $\Delta z_{\mu}(n)$ :

$$
\frac{\partial}{\partial x_{\mu}} \delta\left(x-y-\sum_{k=1}^{N} \Delta z(k)\right)=-\frac{\partial}{\partial \Delta z_{\mu}(N)} \delta\left(x-y-\sum_{k=1}^{N} \Delta z(k)\right) .
$$

Integrating by parts in the expression for $G(x, y)$, one obtains

$$
D_{\mu} G(x, y)=\int_{0}^{\infty} d s e^{-m s}(D z)_{x y} e^{-K} \Phi_{\sigma}(x, y)\left(-\frac{\Delta z_{\mu}(N)}{2 \varepsilon}+O(\sqrt{\varepsilon})\right) .
$$


In the limit $\varepsilon \rightarrow 0, N \rightarrow \infty$ one has $\left.\frac{\Delta z_{\mu}(k)}{2 \varepsilon} \rightarrow \frac{d z_{\mu}(\tau)}{2 d \tau}\right|_{\tau=s}$ and using the relation (4), one obtains

$$
D_{\mu} G(x, y)=-\omega(\tau=s) \frac{d z_{\mu}(t)}{d t} G(x, y)
$$

where the r.h.s. of (B.3) is a symbolic writing implying that $\omega \frac{d z_{\mu}}{d t}$ should be under the integral in $G(x, y)$. Finally, when $G(x, y)$ is expressed via the Hamiltonian $\hat{H}_{\omega}$ (as in the definitions (19) and (21)), then one realizes that $\omega_{i} \dot{z}_{\mu}^{(i)}(t)=p_{\mu}^{(i)}$ and finds the relation:

$$
(m-\hat{D}) G=(m-i \hat{p}) G,
$$

which will be used throughout the paper.

As a check one can see that (B.4) yields the correct form of the free quark propagator in Euclidean space-time.

Consider now a meson in the c.m. system and take into account that for a quark one has

$$
D_{\mu}^{(1)} \Rightarrow-\omega_{1} \dot{z}_{\mu}^{(1)} \Rightarrow i p_{\mu}^{(1)}, \quad D_{\mu}^{(2)}=\omega_{2} \dot{z}_{\mu}^{(2)}=-i p_{\mu}^{(2)},
$$

and $p_{4}^{(1)}=i \omega_{1}, p_{4}^{(2)}=i \omega_{2}$, so that $D_{4}^{(1)} \Rightarrow-\omega_{1}, D_{4}^{(2)} \Rightarrow \omega_{2}$, while with the 3 -momentum $\boldsymbol{p}$, $p_{i}^{(1)}=-p_{i}^{(2)}=p_{i}$

$$
D_{i}^{(1)} \Rightarrow+i p_{i}, \quad D_{i}^{(2)} \Rightarrow i p_{i}
$$

For the factor $Y_{\Gamma}(9)$ one obtains

$$
Y_{\Gamma}=\frac{1}{4} \operatorname{tr}\left(\Gamma\left(m_{1}+\omega_{1} \gamma_{4}-i p_{k} \gamma_{k}\right) \Gamma\left(m_{2}-\omega_{2} \gamma_{4}-i p_{i} \gamma_{i}\right)\right)
$$

Inserting $\Gamma=1, \gamma_{5}, i \gamma_{\mu} \gamma_{5}, \gamma_{\mu}$ in (B.7) one arrives at the expressions (35) for different channels.

\section{APPENDIX C: CALCULATION OF THE PATH INTEGRAL FOR $G_{\Gamma},($ EQ. (8)), IN THE FREE QUARK CASE}

To find the solution in the general case with $W_{\sigma} \neq 1$ and an interaction depending only on the relative quark-antiquark coordinates, we separate here the relative and c.m. coordinates for any path-integral index $k$ as follows:

$$
\begin{aligned}
\Delta \boldsymbol{z}_{1}-\Delta \boldsymbol{z}_{2} & =\boldsymbol{\eta}, \quad \frac{\omega_{1} \Delta \boldsymbol{z}_{1}+\omega_{2} \Delta \boldsymbol{z}_{2}}{\omega_{1}+\omega_{2}}=\boldsymbol{\rho}, \\
\omega_{+} & =\omega_{1}+\omega_{2}, \quad \omega_{\mathrm{r}}=\frac{\omega_{1} \omega_{2}}{\omega_{+}}
\end{aligned}
$$


with the Jacobians:

$$
\begin{aligned}
d^{3} \Delta z_{1} d^{3} z_{2} & =d^{3} \eta d^{3} \rho \\
\frac{d \omega_{1} d \omega_{2}}{\sqrt{\omega_{1} \omega_{2}}} & =\frac{d \omega_{\mathrm{r}}}{\sqrt{\omega_{\mathrm{r}}}} \frac{d \omega_{+}}{2 \sqrt{\omega_{+}-4 \omega_{\mathrm{r}}}}
\end{aligned}
$$

Integrating (8) for $W_{\sigma} \equiv 1$ over $\prod_{k} d \boldsymbol{\rho}(k)$ one obtains

$$
G_{\Gamma}(x)=4 N_{c} Y_{\Gamma} \int \frac{\left(D^{3} \eta\right)_{00}}{l_{\eta}^{3} 4 \bar{\omega}_{1} \bar{\omega}_{2}} \prod_{k} \frac{d \omega_{+}(k)}{\sqrt{\frac{2 \pi}{\Delta t}} 2 \sqrt{\omega_{+}-4 \omega_{\mathrm{r}}(k)}} \frac{d \omega_{\mathrm{r}}(k)}{\sqrt{\omega_{\mathrm{r}}(k) \frac{2 \pi}{\Delta t}}} e^{-\mathcal{F}_{1}}
$$

Here the notation $\left(D^{3} \eta\right)_{a b}$ means the initial $a$ and final $b$ value of relative coordinate; in our case, evidently, for considered current correlator $a=b=0$. Also in Eq. (C.3) we use the notation $l_{\eta}=\left(\frac{2 \pi \Delta t}{\omega_{r}(\bar{k})}\right)^{1 / 2}$. The quantity $\mathcal{F}_{1}$ is defined as

$$
\mathcal{F}_{1}=\sum_{k}\left\{\frac{\omega_{+}(k) \Delta t}{2}+\frac{m^{2} \Delta t}{2 \omega_{\mathrm{r}}(k)}+\frac{\omega_{\mathrm{r}}(k) \eta^{2}(k)}{2 \Delta t}\right\} .
$$

Integration over $d \omega_{+}(k)$ can be easily done,

$$
\prod_{k} \int \frac{d \omega_{+}(k)}{2 \sqrt{\omega_{+}-4 \omega_{\mathrm{r}}(k)}} \frac{\exp \left\{-\sum \omega_{+}(k) \frac{\Delta t}{2}\right\}}{\sqrt{2 \pi \Delta t}}=\prod_{k} 2 e^{-\sum_{k} 2 \omega_{\mathrm{r}}(k) \Delta t} .
$$

Thus at zero c.m. momentum one finds

$$
\int G_{\Gamma}(x) d^{3} \boldsymbol{x}=\int \frac{N_{c} Y_{\Gamma}}{\bar{\omega}_{1} \bar{\omega}_{2}} \frac{\left(D^{3} \eta\right)_{00}}{l_{\eta}^{3}} \prod_{k=1}^{N} \frac{2 d \omega_{\mathrm{r}}(k)}{\sqrt{\frac{\omega_{\mathrm{r}}(k) 2 \pi}{\Delta t}}} e^{-\mathcal{F}_{2}}
$$

where

$$
\mathcal{F}_{2}=\sum_{k}\left\{2 \omega_{\mathrm{r}}(k) \Delta t+\frac{m^{2}}{2 \omega_{\mathrm{r}}(k)} \Delta t+\frac{\omega_{\mathrm{r}}(k)}{2 \Delta t} \eta^{2}(k)\right\} .
$$

At this point one can use the general relation $[30,36]$

$$
\int \frac{\left(D^{3} \eta\right)_{x y}}{l_{\eta}^{3}} e^{-\sum_{k}\left(\frac{\omega_{\mathrm{r}}(k) \eta^{2}(k)}{2 \Delta t}+\hat{V}(k) \Delta t\right)}=\left\langle x\left|e^{-\hat{H} T}\right| y\right\rangle
$$

with

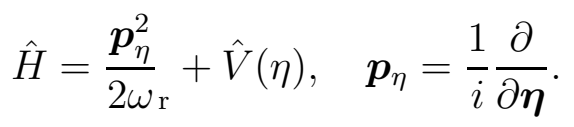

Then for the free case, $\hat{V}(\eta) \equiv 0$, the r.h.s. of (C.8) yields

$$
\left\langle 0\left|\exp \left(-\frac{\boldsymbol{p}^{2}}{2 \omega_{\mathrm{r}}} T\right)\right| 0\right\rangle=\int \frac{d^{3} p}{(2 \pi)^{3}} \exp \left(-\frac{\boldsymbol{p}^{2}}{2 \omega_{\mathrm{r}}} T\right) \text {. }
$$


Integrating first over $d \omega_{\mathrm{r}}(k)$ and performing the steapest descent method (stationary point analysis) one obtains

$$
\int \frac{2 d \omega_{\mathrm{r}}(k)}{\sqrt{\omega_{\mathrm{r}}(k)} \sqrt{\frac{2 \pi}{\Delta t}}} e^{-2 \omega_{\mathrm{r}}(k) \Delta t-\frac{\left(p^{2}+m^{2}\right) \Delta t}{2 \omega_{\mathrm{r}}(k)}}=e^{-2 \sqrt{p^{2}+m^{2}} \Delta t}
$$

and

$$
\int G_{\Gamma}(x) d^{3} x=\int \frac{d^{3} \boldsymbol{p}}{(2 \pi)^{3}} \frac{N_{c} Y_{\Gamma}}{\bar{\omega}_{1} \bar{\omega}_{2}} e^{-2 T \sqrt{\boldsymbol{p}^{2}+m^{2}}} .
$$

For equal current masses $m_{1}=m_{2}=m$ one has evidently $\bar{\omega}_{1}=\bar{\omega}_{2}=2 \omega_{\mathrm{r}}$, and from (C.11) it follows that for the stationary point (for any $k$ ) $2 \omega_{\mathrm{r}}=\sqrt{\boldsymbol{p}^{2}+m^{2}}$.

To compare (C.12) with the standard Feynman amplitude for the free quark loop, one can go to the momentum space,

$$
\begin{aligned}
G_{\Gamma}\left(\boldsymbol{q}=0, q_{4}\right) & =\int_{-\infty}^{\infty} d T e^{i q_{4} T} \int G_{\Gamma}(x) d^{3} x \\
& =\int \frac{d^{3} p}{(2 \pi)^{3}} \frac{N_{c} Y_{\Gamma}}{\boldsymbol{p}^{2}+m^{2}}\left(\frac{1}{2 \sqrt{\boldsymbol{p}^{2}+m^{2}}-i q_{4}}+\frac{1}{2 \sqrt{\boldsymbol{p}^{2}+m^{2}}+i q_{4}}\right) \\
& =\int 4 N_{c} Y_{\Gamma} \frac{d^{3} p}{(2 \pi)^{3} \sqrt{\boldsymbol{p}^{2}+m^{2}}\left[4\left(\boldsymbol{p}^{2}+m^{2}\right)+q_{4}^{2}\right]} \\
& =\int \frac{4 N_{c} Y_{\Gamma} d^{4} p}{(2 \pi)^{4} p^{2}(p-q)^{2}}
\end{aligned}
$$

This result proves our sequence of equations for the free case. For the case of interacting quarks, one should use in (C.8) the spectral decomposition in the infinite set of bound states, as it is done in the main text, Eqs. $(23,25,28)$.

\section{APPENDIX D: THE RELATIVISTIC STRING HAMILTONIAN}

We start with the relativistic $\mathrm{SH} \hat{H}_{\omega}$ for a meson $q_{1} \bar{q}_{2}$, taken in the most general form $[22]:$

$$
\hat{H}_{\omega}=\sum_{i=1,2}\left(\frac{\omega_{i}}{2}+\frac{m_{i}^{2}}{2 \omega_{i}}\right)+\frac{p_{r}^{2}}{2 \omega_{\mathrm{r}}}+V_{\mathrm{GE}}(r)+\int_{0}^{1} d \beta\left(\frac{\sigma^{2} r^{2}}{2 \nu}+\frac{\nu}{2}\right)+\frac{\boldsymbol{L}^{2}}{2 r^{2}} \frac{1}{g\left(\omega_{1}, \omega_{2}, \sigma r\right)} .
$$

Here $\omega_{\mathrm{r}}=\omega_{1} \omega_{2} /\left(\omega_{1}+\omega_{2}\right)$ and $m_{i}(i=1,2)$ are the pole masses of a quark (antiquark) and

$$
g\left(\omega_{1}, \omega_{2}, \sigma r\right)=\omega_{1}(1-\zeta)^{2}+\omega \zeta^{2}+\int_{0}^{1} d \beta(\beta-\zeta)^{2} \nu d \beta
$$


with

$$
\zeta=\frac{\omega_{1}+\frac{1}{2} \sigma r}{\omega_{1}+\omega_{2}+\sigma r} .
$$

In the SH (D.1) taken from [22] we have added the GE potential, $V_{\mathrm{GE}}=-\frac{4}{3} \frac{\alpha_{\mathrm{st}}(r)}{r}$. This can be done due to the property of additivity of the static potential in QCD [39, 40]. $\hat{H}_{\omega}$ depends on the variables $\omega_{1}, \omega_{2}$, and $\nu$, which have been shown to be the canonical variables of the SH [26], and therefore they can be defined from the extremum conditions. But first, instead of the operator $p_{r}^{2}$ in (D.1) we introduce $\boldsymbol{p}^{2}=p_{r}^{2}+\boldsymbol{L}^{2} / r^{2}$ and present $\hat{H}_{\omega}$ as the sum of two terms:

$$
\hat{H}_{\omega}=H_{\omega}^{(0)}+H_{\text {str }},
$$

where

$$
H_{\omega}^{(0)}=\sum_{i=1,2}\left(\frac{\omega_{i}}{2}+\frac{m_{i}^{2}}{2 \omega_{i}}\right)+\frac{\boldsymbol{p}^{2}}{2 \omega_{\mathrm{r}}}+V_{\mathrm{GE}}(r)+\int_{0}^{1} d \beta\left(\frac{\sigma^{2} r^{2}}{2 \nu}+\frac{\nu}{2}\right),
$$

and the "string" part of the $\mathrm{SH}$ is

$$
H_{\mathrm{str}}=-\frac{\boldsymbol{L}^{2}}{2 r^{2} \omega_{\mathrm{r}}}\left[1-\frac{\omega_{\mathrm{r}}}{g\left(\omega_{1}, \omega_{2}, r\right)}\right] .
$$

This term occurs only for the states with $L \neq 0$ and can be considered as a perturbation, because it gives corrections $\lesssim 5 \%$ to the e.v. of the unperturbed Hamiltonian $H_{\omega}^{(0)}$ (D.5). (For light mesons this correction is becoming rather large only for the states with $L \gtrsim 5$ ). Then to determine the variables $\omega_{1}, \omega_{2}$, and $\nu$ we use the following extremum conditions applied to $H_{\omega}^{(0)}$ :

$$
\frac{\partial H_{\omega}^{(0)}}{\partial \omega_{i}}=0, \quad(i=1,2) ; \quad \frac{\partial H_{\omega}^{(0)}}{\partial \nu}=0 .
$$

From (D.7) it follows that

$$
\omega_{i}^{2}=\boldsymbol{p}^{2}+m_{i}^{2}, \quad \nu=\sigma r
$$

and therefore $H_{\omega}^{(0)}$ can be rewritten as

$$
H_{\omega}^{(0)}=\sum_{i=1,2} \sqrt{m_{i}^{2}+\boldsymbol{p}^{2}}+\sigma r+V_{\mathrm{GE}}(r) \equiv T_{R}+V_{0}(r),
$$

where $V_{0}(r)$ is just the same potential as in (14). From (D.9) one can see that the kinetic term $T_{R}$ coincides with the one in the spinless Salpeter equation (SSE). The equation

$$
\left(H_{\omega}^{(0)}+V_{0}(r)\right) \varphi_{n L}(r)=M_{0} \varphi_{n L}(r)
$$


defines the e.v. $M_{0}(n L)$ and e.f. $\varphi_{n L}(r)$. There are two essential differences between our SH $H_{\omega}^{(0)}$ with the kinetic part

$$
T_{R}=\sqrt{m_{1}^{2}+\boldsymbol{p}^{2}}+\sqrt{m_{2}^{2}+\boldsymbol{p}^{2}}
$$

and many other papers where the SSE is used.

The first one is that in (D.11) the quark (antiquark) mass is the pole (current) mass and it is not considered to be a fitting parameter. In Table I we compare the input masses $m_{1}, m_{2}$ used in (D.11) and the constituent masses from [2, 4, 5].

The second difference refers to the string correction $H_{\text {str }}$ which can be rewritten as

$$
H_{\mathrm{str}}=-\frac{\boldsymbol{L}^{2} \sigma}{2 \omega_{\mathrm{r}}}\left\{\frac{g_{1}\left(\omega_{1}, \omega_{2}, r\right)}{r}+\frac{1}{4} \sigma \frac{\left(\omega_{1}-\omega_{2}\right)^{2}}{\left(\omega_{1}+\omega_{2}\right)^{2}} g_{2}\left(\omega_{1}, \omega_{2}, r\right)\right\} .
$$

Here

$$
\begin{aligned}
& g_{1}=\left(\frac{1}{3}-\zeta+\zeta^{2}\right)\left(\omega_{1}+\omega_{2}+\sigma r\right)^{2} \mathcal{F}^{-1}\left(r, \omega_{1}, \omega_{2}\right), \\
& g_{2}=\mathcal{F}^{-1}\left(r, \omega_{1}, \omega_{2}\right)
\end{aligned}
$$

with

$$
\begin{aligned}
\mathcal{F}\left(r_{1}, \omega_{1}, \omega_{2}\right)= & \omega_{1}\left(\omega_{2}+\frac{1}{2} \sigma r\right)^{2}+\omega_{2}\left(\omega_{1}+\frac{1}{2} \sigma r\right)^{2} \\
& +\sigma r\left(\omega_{1}+\omega_{2}+\sigma r\right)^{2}\left(\frac{1}{3}-\zeta+\zeta^{2}\right) .
\end{aligned}
$$

The string correction to a meson mass, $\Delta_{\text {str }}(n L)=\left\langle H_{\text {str }}\right\rangle$, can be calculated with the use of the expressions (D.12-D.14), in which $\omega_{1}, \omega_{2}$ can be replaced by their averaged values with a good accuracy.

For light mesons with $m_{1}=m_{2}=0, \omega_{1}=\omega_{2}=\omega, \sigma\langle r\rangle=2 \omega$, and $\zeta=\frac{1}{2}$, the second term in (D.12) is absent and

$$
\langle\mathcal{F}(r, \omega)\rangle=\frac{32}{3} \omega^{3},\left\langle g_{1}\right\rangle=\frac{1}{8 \omega} ;\left\langle g_{2}\right\rangle=\frac{3}{32 \omega^{3}},
$$

so that in this case $\left(m_{1}=m_{2}=0\right)$ the string correction,

$$
\Delta_{\mathrm{str}}=\left\langle H_{\mathrm{str}}\right\rangle=-\frac{L(L+1)}{\omega} \frac{\sigma\left\langle r^{-1}\right\rangle}{8 \omega},
$$

just coincides with the string correction obtained in [24] for light mesons. It is important that due to the negative sign of the string correction the masses of $P$ - and $D$-wave heavylight mesons appear to be $30-50 \mathrm{MeV}$ smaller in our calculations than in other relativistic models which use the SSE equation [2]. 
TABLE IX: The average energies $\bar{\omega}_{i}(n S)=\left\langle\sqrt{m_{i}^{2}+\boldsymbol{p}^{2}}\right\rangle_{n S}(i=1,2)$, the reduced mass $\omega_{\mathrm{r}}$, and the excitation energy $\varepsilon_{n}\left(\omega_{\mathrm{r}}\right)$ (in $\mathrm{MeV}$ ) for the $1 S$ and $2 S$ heavy-light mesons.

\begin{tabular}{|l|l|l|l|l|l|}
\hline Meson & $D$ & $D_{s}$ & $B$ & $B_{s}$ & $B_{c}$ \\
\hline $\bar{\omega}_{1}(1 S)$ & 507 & 559 & 587 & 639 & 1662 \\
$\bar{\omega}_{2}(1 S)$ & 1509 & 1515 & 4827 & 4830 & 4869 \\
$\omega_{\mathrm{r}}(1 S)$ & 379 & 408 & 523 & 564 & 1238 \\
$\varepsilon_{1}\left(\omega_{\mathrm{r}}\right)$ & 541 & 534 & 432 & 406 & 149 \\
\hline $\bar{\omega}_{1}(2 S)$ & 643 & 692 & 741 & 789 & 1732 \\
$\bar{\omega}_{2}(2 S)$ & 1585 & 1590 & 4862 & 4865 & 4898 \\
$\omega_{\mathrm{r}}(2 S)$ & 457 & 482 & 643 & 679 & 1279 \\
$\varepsilon_{2}\left(\omega_{\mathrm{r}}\right)$ & 1164 & 1124 & 985 & 959 & 687 \\
\hline
\end{tabular}

TABLE X: The w.f. at the origin $\left|R_{n}(0)\right|^{2}\left(\right.$ in $\left.\mathrm{GeV}^{3}\right)$ and $\left\langle\boldsymbol{p}^{2}\right\rangle_{n S}\left(\right.$ in $\left.\mathrm{GeV}^{2}\right)$ in einbein approximation for the $1 S$ and $2 S$ states of heavy-light mesons.

\begin{tabular}{|l|ll|ll|}
\hline \multirow{2}{*}{ Meson } & \multicolumn{2}{|c|}{$1 S$} & \multicolumn{2}{c|}{$2 S$} \\
\cline { 2 - 5 } & $\left|R_{1}(0)\right|^{2}$ & $\left\langle\boldsymbol{p}^{2}\right\rangle_{1 S}$ & $\left|R_{2}(0)\right|^{2}$ & $\left\langle\boldsymbol{p}^{2}\right\rangle_{2 S}$ \\
\hline$D$ & 0.272 & 0.273 & 0.266 & 0.464 \\
$D_{s}$ & 0.291 & 0.290 & 0.284 & 0.482 \\
$B$ & 0.410 & 0.359 & 0.410 & 0.599 \\
$B_{s}$ & 0.455 & 0.383 & 0.439 & 0.624 \\
$B_{c}$ & 1.470 & 0.784 & 1.032 & 1.023 \\
\hline
\end{tabular}

In Table IX we give the values of the average kinetic energies $\bar{\omega}_{1}$ and $\bar{\omega}_{2}$, and the excitation energy $\varepsilon\left(\omega_{\mathrm{r}}\right)$, which are needed to determine the self-energy contribution (32) to $M_{\operatorname{cog}}(n S)$.

To calculate the decay constants $f_{\mathrm{P}}$ and $f_{\mathrm{V}}$ we need also to know the w.f. at the origin $\varphi_{n}(0)=R_{n}(0) / \sqrt{4 \pi}$ and the m.e. $\left\langle\boldsymbol{p}^{2}\right\rangle_{n S}$. Their values are given in Table $\mathrm{X}$.

As seen from Table IX the "constituent" masses $\omega_{1}(2 S)$ and $\omega_{1}(1 S)$ of the lighter quark $q_{1}\left(\right.$ or $\left.\bar{q}_{1}\right)$ differ by $\sim 130 \mathrm{MeV}$ for the $D$ and $D_{s}$ mesons and $\sim 150 \mathrm{MeV}$ for the $B$ and 
$B_{s}$ mesons. This difference in $\omega_{1}(n S)$ illustrates the statement that in relativistic approach there does not exist a universal constituent mass $\omega_{i}$ for different $n L$ states, but only the pole (current) quark masses may be considered as universal input.

At this point we remind that the expressions $(28,35)$ for the decay constants have been derived in the EA approximation and therefore to have a consistent description one needs to take also $\left|R_{n}(0)\right|^{2}$ and $\left\langle\boldsymbol{p}^{2}\right\rangle_{n S}$ as calculated in the EA. In this approximation, instead of the extremum conditions (D.7), different conditions may be used, [26], namely,

$$
\frac{\partial M^{\mathrm{EA}}}{\partial \tilde{\omega}_{1}}=0, \quad \frac{\partial M^{\mathrm{EA}}}{\partial \tilde{\omega}_{2}}=0
$$

where in the EA we use the notations $\tilde{\omega}_{1}$ and $\tilde{\omega}_{2}$ instead of $\bar{\omega}_{1}$ and $\bar{\omega}_{2}$. The mass $M^{\mathrm{EA}}(n L)$ satisfies the equation,

$$
H_{0}^{\mathrm{EA}} \tilde{\varphi}_{n L}(r)=M_{0}^{\mathrm{EA}}(n L) \tilde{\varphi}_{n L}(r)
$$

where the EA Hamiltonian [26] is given by,

$$
H_{0}^{\mathrm{EA}}=\sum_{i=1,2}\left(\frac{\tilde{\omega}_{i}}{2}+\frac{m_{i}^{2}}{2 \tilde{\omega}_{i}}\right)+\frac{\boldsymbol{p}^{2}}{2 \omega_{\mathrm{r}}}+V_{0}(r),
$$

and has the same interaction $V_{0}(r)$ and the reduced mass is given by $\tilde{\omega}_{\mathrm{r}}=\left(\tilde{\omega}_{1} \tilde{\omega}_{2}\right) /\left(\tilde{\omega}_{1}+\tilde{\omega}_{2}\right)$. Then writing

$$
M^{\mathrm{EA}}(n L)=\sum_{i=1,2}\left(\frac{\tilde{\omega}_{i}}{2}+\frac{m_{i}^{2}}{2 \tilde{\omega}_{i}}\right)+\varepsilon_{n L}\left(\omega_{r}\right),
$$

the excitation energy $\varepsilon\left(\omega_{r}\right)$ satisfies the equation

$$
\left\{\frac{\boldsymbol{p}^{2}}{2 \tilde{\omega}_{\mathrm{r}}}+V_{0}(r)\right\} \tilde{\varphi}_{n L}(r)=\varepsilon_{n L}\left(\omega_{\mathrm{r}}\right) \tilde{\varphi}_{n L}(r) .
$$

With the use of (D.20) the extremum conditions (D.17) reduce to the equations:

$$
\tilde{\omega}_{i}^{2}=m_{i}^{2}-2 \tilde{\omega}_{r}^{2} \frac{\partial \varepsilon}{\partial \tilde{\omega}_{\mathrm{r}}} \quad(i=1,2) .
$$

The derivative $\partial \varepsilon\left(\tilde{\omega}_{\mathrm{r}}\right) / \partial \tilde{\omega}_{\mathrm{r}}$ in (D.22) is a very smooth function of the variable $\tilde{\omega}_{\mathrm{r}}(n L)$ and can be easily calculated. Equation (D.21) formally coincides with the Schrödinger equation but differs from the physical point of view: the masses $\tilde{\omega}_{i}(n L)(i=1,2)$ and $\left.\tilde{\omega}_{\mathrm{r}}(n L)\right)$, being the average kinetic energy of a quark (antiquark), are different for every $n L$ state. In this way relativistic corrections are taken into account in Eq. (D.21)) through the increase of $\tilde{\omega}_{1}$ and $\tilde{\omega}_{2}$. 
In the EA the values of $\tilde{\omega}_{i}$ appear to be very close to $\bar{\omega}_{i}$, determined from the SSE (26) or (D.10). Therefore one can define $\omega_{i}(n L)$, solving the SSE once and calculating the m.e.

$$
\bar{\omega}_{i}(n L)=\left\langle\sqrt{\boldsymbol{p}^{2}+m^{2}}\right\rangle_{n L},
$$

instead of calculations of a variety of m.e. in the solutions of (D.21) for different $n L$ states. However, even for $\tilde{\omega}_{i}=\omega_{i}$ the e.v. $M_{0}^{\mathrm{EA}}(n L)$ (D.18) slightly differs from $M_{0}(n L)$ for the SSE with the same quark pole mass $m_{q}$. The differences between them may be called the relativistic correction $\delta_{R}$ :

$$
M_{0}(n L) \equiv M_{0}^{\mathrm{EA}}(n L)-\delta_{R},
$$

which can be approximately calculated,

$$
\delta_{R} \approx \frac{\left\langle\boldsymbol{p}^{2}\right\rangle_{R}+m_{1}^{2}-\omega_{1}^{2}}{2 \omega_{1}} .
$$

In (D.25) the index "1" refers to the lighter quark (antiquark), its pole mass $m_{1}$ and kinetic energy $\omega_{1}$ (D.23). Such a difference between $M_{0}$ and $M_{0}^{\mathrm{EA}}$ occurs due to the fact that

$$
\sqrt{\left\langle\boldsymbol{p}^{2}+m_{i}^{2}\right\rangle_{n L}} \neq\left\langle\sqrt{\boldsymbol{p}^{2}+m_{i}^{2}}\right\rangle_{n L} .
$$

Surprisingly, for the $D$ and $D_{s}$ and $B$ and $B_{s}$ mesons $\delta_{R}$ remains almost constant, being approximately equal to $70 \mathrm{MeV}$ for the ground states and about $100 \mathrm{MeV}$ for the $2 \mathrm{~S}$ states. For the heavy $B_{c}$ meson $\delta_{R} \approx 20 \mathrm{MeV}$ is essentially smaller.

\section{APPENDIX E: THE SELF-ENERGY CORRECTION TO THE MESON MASS}

The self-energy correction to the meson mass originates from the NP contribution to the squared quark mass $m_{q}^{2}$ as a result of the spin interaction of a quark with NP background gluonic field [35]. It was shown that only due to the presence of this correction in the meson mass it is possible to obtain a linear Regge trajectory for light mesons [24]. With the use of the old result from [35] and the recent result from [54] it can be presented as

$$
\Delta_{\mathrm{SE}}(n L)=\sum_{i=1,2}\left(-\frac{1.5 \sigma \eta_{f}^{i}}{\pi \bar{\omega}_{i}}+\frac{\sigma^{2}}{4 \bar{\omega}_{i}\left[m+\bar{\omega}_{i} / 2+\varepsilon\left(\omega_{\mathrm{r}}\right)\right]^{2}}\right) .
$$

Here $m_{i}(i=1,2)$ is the pole mass of $i$-th quark (antiquark), $\bar{\omega}_{i}$ is determined by (D.7), $\omega_{\mathrm{r}}$ is the reduced mass, $\varepsilon\left(\omega_{\mathrm{r}}\right)$ is defined by the solution of the equation (D.21). The factor $\eta_{f}^{i}$ 
depends on the flavor of a given quark (antiquark) $q_{i}\left(\bar{q}_{i}\right)$. The first, negative term in (E.1) was calculated in the Simonov's paper [35] while the second, positive term is rather small and has been derived recently [54]. The analytical expression for $\eta_{f}^{i}$ differs for a heavy quark with mass $m_{q}>T_{g}^{-1}$ and $m_{q}<T_{g}^{-1}$, where $T_{g}$ is the gluonic (vacuum) correlation length which defines the behaviour of the bilocal vacuum correlators $D(x)$ and $D_{1}(x)$ [21]. The value $T_{g}$ has been measured in lattice QCD and in the quenched approximation $T_{g} \lesssim 0.2 \mathrm{fm}$ [44] while in the unquenched case $T_{g}$ is larger $\left(T_{g} \approx 0.30 \mathrm{fm}\right)$ [45].

Introducing the variable $y=m_{q} T_{g}$ (for $c, b$ quarks $y>1$ and for $u(d), s$ quarks $y<1$ ) $\eta_{f}$ is given by the following expressions [35]:

$$
\begin{aligned}
& \eta_{f}=\frac{1+2 y^{2}}{\left(y^{2}-1\right)^{2}}-\frac{3 y^{2}}{\left(y^{2}-1\right)^{5 / 2}} \arctan \sqrt{y^{2}-1} \quad \text { for } y \geq 1, \\
& \eta_{f}=\frac{1+2 y^{2}}{\left(1-y^{2}\right)^{2}}-\frac{3 y^{2}}{\left(1-y^{2}\right)^{5 / 2}} \ln \frac{1+\sqrt{1-y^{2}}}{y} \quad \text { for } y<1 .
\end{aligned}
$$

For $m_{u}, m_{d} \rightarrow 0$ the quantity $\eta_{f}$ tends to 1 , while for $m_{q} \rightarrow \infty$ the value $\eta_{f} \rightarrow 0$. Here for $m_{u}=m_{d} \approx 0$ and $m_{b}=4.78 \mathrm{GeV}$ we have

$$
\begin{aligned}
& \eta_{f}=1.0, \quad \text { for } u(d) \text { quarks } \\
& \eta_{b}=0.03, \quad \text { for } b \text { quarks. }
\end{aligned}
$$

However, since the value of $T_{g}$ and the mass of the $s$ quark are not known with a good accuracy, the value of $\eta_{s}$ may essentially differ. Then from (E.2) we obtain

$$
\begin{aligned}
& \eta_{s}=0.87 \text { for } y=0.20\left(T_{g}=0.22 \mathrm{fm}, m_{s}=0.18 \mathrm{GeV}\right), \\
& \eta_{s}=0.76 \text { for } y=0.33\left(T_{g}=0.32 \mathrm{fm}, m_{s}=0.22 \mathrm{GeV}\right) .
\end{aligned}
$$

and also for $c$ quarks

$$
\eta_{c}=0.36\left(T_{g}=0.2 \mathrm{fm}, m_{c}=1.4 \mathrm{GeV}\right) .
$$

In our analysis here we use the following numbers:

$$
\eta_{u(d)}=1.0, \eta_{s}=0.65, \eta_{c}=0.35, \eta_{b}=0.025 .
$$

The contribution of the $b$ quark to $\left.\Delta_{\mathrm{SE}}(\mathrm{E} .1)\right)$ is about $-1 \mathrm{MeV}$ and can be neglected.

Finally, we notice that in the first term in (E.1) we use the number 1.5 instead of the number 2.0 derived in [35] (where $\eta_{u(d)}=0.9$ was taken, as well as in [24]). This change is 
made because here we do not neglect the contribution of the correlator $D_{1}(x)$ as in the first paper of [35]. Instead at $x=0$ we use the relation $D(0)+D_{1}(0)=\frac{\pi^{2}}{18} G_{2}$, where $G_{2}$ is the gluonic condensate (the details are given in second paper of [54]).

\section{ACKNOWLEDGMENTS}

This work is supported by the Federal Program of the Russian Ministry of Industry, Science, and Technology No.40.052.1.1.1112, and by the grant of RFBR No. 06-02-17012 and State Contract No 02.445.11.7424.

[1] H. Krasemann, Phys. Lett. B 96, 397 (1980); D. Silverman and H. Yao, Phys. Rev. D 38, 214 (1988).

[2] S. Godfrey, N. Isgur, Phys. Rev. D 32, 189 (1985); S. Godfrey, Phys. Rev. D 70, 054017 (2004) and references therein.

[3] V.V. Kiselev, A.E. Kovalsky, A.K. Likhoded, Nucl. Phys. B 585, 353 (2002); S.S.Gershtein et al., Phys. Usp. 38, 1 (1995); Phys. Rev. D51, 3613 (1995).

[4] M. Di Pierro, E. Eichten, Phys. Rev. D 64, 114004 (2001).

[5] Guo-Li Wang, Phys. Lett. B 633, 492 (2006).

[6] D. Ebert, R.N. Faustov and V.O. Galkin, Phys. Lett. B 635, 93(2006); Mod. Phys. Lett. A 17, 803 (2002); V.O. Galkin, A.Yu. Mishurov and R.N. Faustov, Sov. J. Nucl. Phys. 53, 1026 (1991) [Yad. Fiz. 53, 1676 (1991)].

[7] G. Cvetič, C.S. Kim, G.-L. Wang, and W. Namgung, Phys. Lett. B 596, 84 (2004).

[8] J. He, B. Julia-Diaz and Y.B. Dong, Eur. Phys. J A 24, 411 (2005); P. Maris and C.D. Roberts, Int. J. Mod. Phys. E 12, 297 (2003); P. Maris and P.C. Tandy, Phys. Rev. C 60, 055214 (1999); Phys. Rev. C 62, 055204 (2000); M. Koll et al., Eur. Phys. J. A 9, 73 (2000).

[9] S.Narison, hep-ph/0202200; Phys. Lett. B 520, 115 (2001); ibid 322, 247 (1994); ibid 198, 104 (1987).

[10] A.A. Penin and M. Steinhauser, Phys. Rev. D 65, 054006 (2002); M. Jamin, B.O. Lange, Phys. Rev. D 65, 056005 (2002) and reference therein.

[11] For recent reviews see T. Onogi, hep-lat/0610115; 
[12] A. Ali Khan et al., hep-lat/0701015; Phys. Rev. D 65, 054505 (2002); ibid 67, 059901(E) (2003); ibid 64, 054504 (2001).

[13] C. Aubin et al., Phys. Rev. Lett. 95, 122002 (2005); Phys. Rev. D 70, 114501 (2004); M. Wingate et al., Phys. Rev. Lett. 92, 022001 (2004); A. Gray et al., Phys. Rev. Lett. 95, $212001(2005)$.

[14] T.W. Chiu et al., Phys. Lett. B 624, 31 (2005); A. Jüttner and J. Rolf, Phys. Lett. B 560, 59 (2003); (ALPHA Collaboration) J. Rolf et al., Nucl. Phys. Proc. Suppl. 129, 322 (2004); hep-lat/0309072.

[15] P. Zweber, hep-ex/0701018 and references therein.

[16] Particle Data Group, S. Eidelman et al., Phys. Lett. B 592, 1 (2004).

[17] (CLEO Collaboration) M. Artuso et al., hep-ex/0607074;Phys. Rev. Lett. 95, 251801 (2005); (CLEO Collaboaration) G. Bonvicini et al., Phys. Rev. D 70, 112004 (2004).

[18] (BELLE Collaboration) K. Ikado et al., hep-ex/0604018; (BaBar Collaboration) B. Aubert et al., hep-ex/0608019.

[19] R. Mommsen, hep-ex/0612003; (CLEO Collaboration) G. Bonvicini et al., Phys. Rev. Lett. 96, 022002 (2006); (CDF Collaboraton) A.Abulencia et al. Phys. Rev. Lett. 96, 082002 (2006); O.Aquinas et al., (CLEO Collaboration) Phys. Rev. Lett. 96, 152001 (2006).

[20] Yu.A. Simonov, Z. Phys. C 53, 419 (1992).

[21] A. Di Giacomo, H.G. Dosch, V.I. Shevchenko, Yu.A. Simonov, Phys. Rep. 372, 319 (2002); Yu.A. Simonov, Phys. Atom Nucl. 67, 846; 1027 (2004); hep-ph/0302090, hep-ph/0305281.

[22] A.Yu. Dubin, A.B.Kaidalov, Yu.A.Simonov, Phys. Atom. Nucl. 56 , 1795 (1993) [Yad. Fiz. 56, 2137 (1993)]; hep-ph/9911237; A.Yu. Dubin, A.B.Kaidalov, Yu.A.Simonov, Phys. Lett. B323, 41 (1994).

[23] H.G. Dosch, Phys. Lett. B 190, 177 (1987); Yu.A. Simonov, Nucl. Phys. B 307, 512 (1988); H.G. Dosch and Yu.A. Simonov, Phys. Lett. B 205, 339 (1988).

[24] A.M. Badalian, B.L.G. Bakker, Yu.A. Simonov, Phys. Rev. D 66, 034026 (2002); A.M. Badalian, B.L.G. Bakker, Phys. Rev. D 66 (2002) 034025.

[25] A.M. Badalian, V.L. Morgunov, Phys. Rev. D 60,116008 (1999); A.M. Badalian, B.L.G. Bakker, Phys. Rev. D 62, 094031 (2000).

[26] Yu.S. Kalashnikova, A.V. Nefediev, Yu.A. Simonov, Phys. Rev. D 64, 014037 (2001); Yu.S. Kalashnikova and A.V.Nefediev, Phys. Lett. B 530, 117 (2002); A.V. Nefediev, JETP Lett. 
78, 349 (2003); Yu.S. Kalashnikova, A.V. Nefediev, Phys. At. Nucl. 60, 1389 (1997); ibid. 61, 785 (1998).

[27] Yu.A. Simonov, Phys. Atom Nucl. 67, 1027 (2004) [Yad. Fiz. 67, 1050 (2004); Yu.A. Simonov (in preparation).

[28] Yu.A. Simonov, Phys. Atom. Nucl 60, 2069 (1997), hep-ph/9704301; Yu.A. Simonov and J.A. Tjon, Phys. Rev. D 62, 014501 (2000), ibid 62, 094511 (2000); Yu.A. Simonov, Phys. Rev. D 65, 094018 (2002).

[29] V.A. Fock, Izv. Akad. Nauk USSR, OMEN (1937), 557; J. Schwinger, Phys. Rev. 82, 664 (1951); R.P. Feynman, Phys. Rev. 80, 440 (1950); ibid. 84, 108 (1951).

[30] Yu.A. Simonov, J.A. Tjon, Ann. Phys. 228, 1 (1993); ibid 300, 54 (2002).

[31] L. Brink, P. Di Vecchia, P. Howe, Nucl. Phys. B 118, 76 (1977); A. Polyakov, Gauge fields and strings, Harwood Ac. Publ., 1987.

[32] B.S. De Witt, Phys. Rev. 162, 1195, 1239 (1967); J. Honerkamp, Nucl. Phys. B 48, 269 (1972); G.'t Hooft Nucl. Phys. B 62, 444 (1973), Lectures at Karpacz, in: Acta Univ. Wratislaviensis 368, 345 (1976); L.F. Abbot, Nucl. Phys. B 185, 189 (1981).

[33] Yu.A. Simonov, in: Lecture Notes in Physics, v.479, p.144, Springer, 1996; Yu.A. Simonov, Phys. At. Nucl. 58, 107 (1995) [Yad. Fiz. 58, 113 (1995)]; hep-ph/9311247.

[34] Yu.A. Simonov, in Proceedings of the XVII Autumn School, Lisboa, Portugal, 29 Sept.- 4 Oct. Eds. L. Ferreira, P. Nogueira and J. Silva-Marco (World Scientific, Singapore, 2000) p.60; [arXiv:hep-ph/9911237].

[35] Yu.A. Simonov, Phys. Lett. B 515, 137 (2001); hep-ph/0105141, see Err. in A. Di Giacomo, Yu.A. Simonov, Phys. Lett. B 595, 368 (2004).

[36] R.P. Feynman, A.R. Hibbs, Quantum Mechanics and Path Integrals, McGraw-Hill, N.Y., 1965.

[37] V.L. Morgunov, A.V. Nefediev and Yu.A. Simonov, Phys. Lett. B 459, 653 (1999).

[38] A.M. Badalian, A.I. Veselov, B.L.G. Bakker, Phys. Atom. Nucl. 67, 1367 (2004); hepph/0311010; A.M. Badalian, A.I. Veselov, B.L.G. Bakker, J. Phys. G 31, 417 (2005).

[39] A.M. Badalian, D.S. Kuzmenko, Phys. Rev.D 65, 016004 (2002).

[40] G. Bali, Phys. Lett. B 460, 170 (1999).

[41] (CDF Collaboration) A. Abulencia et al., Phys. Rev. Lett. 96, 082002 (2006); hep-ex/0505076; (CDF and D0 Collaborations) P. Catastini, hep-ph/0605051.

[42] J. Pantaleone, S.-H.H. Tye, and Y.J. Ng, Phys. Rev. D 33, 777 (1986). 
[43] A.M. Badalian, B.L.G. Bakker, Phys.Atom. Nucl. 69, 734 (2006); Phys. Rev. D64, 114010 (2001).

[44] A. Di Giacomo, H. Panagopoulos, Phys. Lett. B 285, 133 (1992); G.S. Bali, N. Brambilla, A. Vairo, Phys. Lett. B 421, 265 (1998).

[45] M. D’Elia, A. Di Giacomo, E. Meggiolaro, Phys. Lett. B 408, 315 (1997).

[46] A.M. Badalian, B.L.G. Bakker, Phys. Rev. D 67, 071901 (2003).

[47] V.V. Kiselev, Central Eur. J. Phys. 2, 523 (2004).

[48] A. Le Yaouanc, et al., Z. Phys. C 28,309 (1985); Yu.A. Simonov, Phys. At. Nucl. 64, 1876 (2001).

[49] Yu.S. Kalashnikova, D.S. Kuzmenko, Phys. At. Nucl. 67, 538 (2004), ibid 66, 955 (2003).

[50] A.B. Kaidalov, Yu.A. Simonov, Phys. Lett. B 477, 163 (2000); ibid. 636101 (2006).

[51] A.M. Badalian, B.L.G. Bakker, hep-ph/0604243.

[52] M.A. Shifman, M.B. Voloshin, J. Nucl. Phys. 45, 292 (1987).

[53] K.G. Chetyrkin, A.G. Grozin, Nucl. Phys. B 666, 289 (2003).

[54] Yu.A. Simonov, Phys. Atom Nucl. 68, 709 (2005) [Yad. Fiz. 68, 739 (2005)], hep-ph/0407027. 